\title{
Symmetries of curved superspace in five dimensions
}

\author{
Sergei M. Kuzenko, Joseph Novak and Gabriele Tartaglino-Mazzucchelli \\ School of Physics M013, The University of Western Australia, \\ 35 Stirling Highway, Crawley W.A. 6009, Australia \\ E-mail: sergei.kuzenko@uwa.edu.au, joseph.novak@uwa.edu.au, \\ gabriele.tartaglino-mazzucchelli@uwa.edu.au
}

ABSTRACT: We develop a formalism to construct supersymmetric backgrounds within the superspace formulation for five-dimensional (5D) conformal supergravity given in arXiv:0802.3953. Our approach is applicable to any off-shell formulation for 5D minimal Poincaré and anti-de Sitter supergravity theories realized as the Weyl multiplet coupled with two compensators. For those superspace backgrounds which obey the equations of motion for (gauged) supergravity, we naturally reproduce the supersymmetric solutions constructed a decade ago by Gauntlett et al. For certain supersymmetric backgrounds with eight supercharges, we construct a large family of off-shell supersymmetric sigma models such that the superfield Lagrangian is given in terms of the Kähler potential of a real analytic Kähler manifold.

KEYwords: Extended Supersymmetry, Superspaces, Supergravity Models, Space-Time Symmetries

ARXIV EPRINT: 1406.0727 


\section{Contents}

1 Introduction 2

2 The Weyl multiplet in superspace $\quad 3$

3 (Conformal) isometries $\quad 6$

3.1 Conformal isometries 6

3.2 Conformally related superspaces 9

3.3 Isometries 9

4 Supersymmetric backgrounds: general formalism 10

$\begin{array}{lll}4.1 & \text { Conformal Killing spinors } & 11\end{array}$

4.2 Killing spinors 13

5 Supersymmetric backgrounds: eight supercharges $\quad 14$

$\begin{array}{lll}5.1 & \text { The case } s \neq 0 & 14\end{array}$

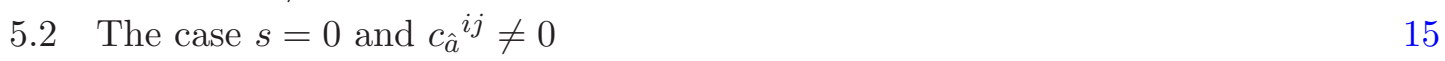

$\begin{array}{lll}5.3 & \text { The case } s=0 \text { and } c_{\hat{a}}^{i j}=0 & 17\end{array}$

6 Vector multiplet compensator $\quad \mathbf{2 0}$

6.1 Supersymmetric backgrounds 21

6.2 The dilaton Weyl multiplet 21

$\begin{array}{lll}7 & \mathcal{O}(2) & \text { multiplet compensator }\end{array}$

7.1 Supersymmetric backgrounds 23

$\begin{array}{lll}7.2 & \text { Supersymmetric backgrounds with eight supercharges } & 23\end{array}$

8 Off-shell supergravity $\quad 23$

8.1 Supersymmetric backgrounds 24

8.2 Supersymmetric backgrounds with eight supercharges 25

$\begin{array}{ll}\text { 8.2.1 The case } s \neq 0 & 25\end{array}$

$\begin{array}{ll}\text { 8.2.2 The case } s=0 & 25\end{array}$

9 Supersymmetric solutions in Poincaré and anti-de Sitter supergravities 26

10 Concluding comments $\quad 28$

$\begin{array}{ll}\text { A (Conformal) isometries in curved space } & 31\end{array}$

B Conformal Killing spinors and bilinears $\quad 34$ 


\section{Introduction}

Six years ago, two of us developed the superspace approach to off-shell $\mathcal{N}=1$ supergravitymatter couplings in five dimensions (5D) $[1-3] .{ }^{1}$ As concerns the Weyl multiplet of 5D conformal supergravity, its formulation given in [3] may be simply thought of as an alternative realization of the one discovered a few years earlier within the component superconformal tensor calculus [7-12]. ${ }^{2}$ However, the real power of the superspace approach of [1-3] is that it offers a generating formalism to realize the most general locally supersymmetric $\sigma$-model couplings and hence, in principle, to construct new quaternionic Kähler metrics. This is achieved by making use of the concept of covariant projective supermultiplets [1-3]. These supermultiplets are a curved-superspace extension of the so-called superconformal projective multiplets [15], which in the $4 \mathrm{D} \mathcal{N}=2$ super-Poincaré case reduce to the offshell projective multiplets pioneered by Lindström and Roček [16, 17]. Among the most interesting covariant projective supermultiplets are polar ones that have infinitely many auxiliary fields. Such off-shell supermultiplets are practically impossible to engineer or to deal with in the framework of superconformal tensor calculus. This is why they had never appeared within the component settings of [4-12], which deal only with hypermultiplets either with a gauged central charge [4-9] or that are on-shell [10-12].

The superspace formulation developed in [1-3] provides a universal setting to generate off-shell supersymmetric field theories on curved backgrounds. For instance, the general 5D $\mathcal{N}=1$ rigid supersymmetric theories in $\mathrm{AdS}_{5}$, which were constructed in [18], can easily be read off from the supergravity-matter systems proposed in [1-3] by properly freezing the supergravity fields. Of course, the problem of constructing supersymmetric field theories on a given spacetime is well formulated only if this manifold is a supersymmetric background, i.e. it admits rigid supersymmetries. Thus one is naturally led to the more general problem of looking for those curved superspaces that possess (conformal) isometries. In the case of $4 \mathrm{D} \mathcal{N}=1$ old minimal supergravity, the latter problem was addressed in [19] almost twenty years ago. The approach presented in [19] is universal, for in principle it may be generalized to supersymmetric backgrounds associated with any supergravity theory formulated in superspace. In particular, it has already been used to construct rigid supersymmetric field theories in 5D $\mathcal{N}=1[18], 4 \mathrm{D} \mathcal{N}=2[20-23]$ and $3 \mathrm{D}(p, q)$ anti-de Sitter [24-26] superspaces.

Recently, a number of publications have appeared devoted to the construction of supersymmetric backgrounds associated with off-shell supergravity theories in diverse dimensions, see [27-44] and references therein. Inspired by [27], these works used component field considerations. In the case of $4 \mathrm{D} \mathcal{N}=1$ supergravity, it was shown [45] how to derive

\footnotetext{
${ }^{1}$ In five dimensions, different authors use different notations, $\mathcal{N}=1$ or $\mathcal{N}=2$, for supersymmetric theories with eight supercharges. The notation $\mathcal{N}=1$ is used, e.g., in refs. [1-3]. The rationale for its use is that the case of eight supercharges corresponds to simple supersymmetry. The alternative notation $\mathcal{N}=2$ is used, e.g., in [4-12]. The reason for this choice is that dimensional reduction of five-dimensional theories with eight supercharges leads to $\mathcal{N}=2$ theories in four dimensions.

${ }^{2}$ The minimal multiplet of $5 \mathrm{D} \mathcal{N}=1$ supergravity was originally sketched, within a superspace setting, by Howe in 1981 [13] (using the supercurrent multiplet constructed in [14]) and fully elaborated in [1, 2]. It was re-discoverd by Zucker [4-6] who elaborated on the component implications of [13].
} 
the key component results of, e.g., [27, 34] from the more general superspace construction of [19]. Recently, the formalism of [45] was extended to construct supersymmetric backgrounds [46] associated with all known off-shell formulations for $3 \mathrm{D} \mathcal{N}=2$ supergravity $[24,47]$. The results obtained are in agreement with the component considerations of $[38,39,43]$. In the present paper, we apply the ideas and techniques developed in $[45,46]$ to construct supersymmetric backgrounds associated with 5D $\mathcal{N}=1$ supergravity.

This paper is organized as follows. Section 2 contains a brief review of the superspace formulation for $5 \mathrm{D}$ conformal supergravity [3]. In section 3 we study (conformal) isometries of a background superspace. In section 4 we study bosonic backgrounds that possess at least one (conformal) Killing spinor. Maximally supersymmetric backgrounds are described in section 5. Sections 6 and 7 are concerned with additional restrictions on the background geometry, which arise when a single conformal compensator, a vector multiplet or an $\mathcal{O}(2)$ multiplet, is turned on. Section 8 is devoted to supersymmetric backgrounds in off-shell supergravity. Supersymmetric solutions in Poincaré and anti-de Sitter supergravity theories are studied in section 9. Finally, concluding comments are given in section 10.

The main body of the paper is accompanied by two technical appendices. In appendix A we recall how the problem of computing the (conformal) isometries of a curved spacetime is addressed within the Weyl-invariant formulation for gravity. In appendix B we discuss the properties of bilinears constructed from a conformal Killing spinor.

\section{The Weyl multiplet in superspace}

In this section we briefly review the superspace description [3] of the Weyl multiplet of 5D conformal supergravity. Our notation and conventions follow those introduced in [48] (see also the appendix of [2]).

Let $z^{\hat{M}}=\left(x^{\hat{m}}, \theta_{i}^{\hat{\mu}}\right)$ be local bosonic $(x)$ and fermionic $(\theta)$ coordinates parametrizing a curved five-dimensional superspace $\mathcal{M}^{5 \mid 8}$, where $\hat{m}=0,1, \cdots, 4, \hat{\mu}=1, \cdots, 4$, and $i=\underline{1}, \underline{2}$. The Grassmann variables $\theta_{i}^{\hat{\mu}}$ are assumed to obey the standard pseudo-Majorana reality condition $\left(\theta_{i}^{\hat{\mu}}\right)^{*}=\theta_{\hat{\mu}}^{i}=\varepsilon_{\hat{\mu} \hat{\nu}} \varepsilon^{i j} \theta_{j}^{\hat{\nu}}$. The tangent-space group is chosen to be $\mathrm{SO}(4,1) \times \mathrm{SU}(2)$ and the superspace covariant derivatives $\mathcal{D}_{\hat{A}}=\left(\mathcal{D}_{\hat{a}}, \mathcal{D}_{\hat{\alpha}}^{i}\right)$ have the form

$$
\mathcal{D}_{\hat{A}}=E_{\hat{A}}+\Omega_{\hat{A}}+\Phi_{\hat{A}} \text {. }
$$

Here $E_{\hat{A}}=E_{\hat{A}}^{\hat{M}}(z) \partial_{\hat{M}}$ is the (inverse) supervielbein, with $\partial_{\hat{M}}=\partial / \partial z^{\hat{M}}$,

$$
\Omega_{\hat{A}}=\frac{1}{2} \Omega_{\hat{A}}^{\hat{b} \hat{c}} M_{\hat{b} \hat{c}}=\Omega_{\hat{A}}^{\hat{\beta} \hat{\gamma}} M_{\hat{\beta} \hat{\gamma}}, \quad M_{\hat{a} \hat{b}}=-M_{\hat{b} \hat{a}}, \quad M_{\hat{\alpha} \hat{\beta}}=M_{\hat{\beta} \hat{\alpha}}
$$

is the Lorentz connection, and

$$
\Phi_{\hat{A}}=\Phi_{\hat{A}}^{k l} J_{k l}, \quad J_{k l}=J_{l k}
$$

is the $\mathrm{SU}(2)$ connection. The Lorentz generators with vector indices $\left(M_{\hat{a} \hat{b}}\right)$ and spinor indices $\left(M_{\hat{\alpha} \hat{\beta}}\right)$ are related to each other by the rule: $M_{\hat{a} \hat{b}}=\left(\Sigma_{\hat{a} \hat{b}}\right)^{\hat{\alpha} \hat{\beta}} M_{\hat{\alpha} \hat{\beta}}$. The generators 
of $\mathrm{SO}(4,1) \times \mathrm{SU}(2)$ act on the covariant derivatives as follows: ${ }^{3}$

$$
\left[J^{k l}, \mathcal{D}_{\hat{\alpha}}^{i}\right]=\varepsilon^{i(k} \mathcal{D}_{\hat{\alpha}}^{l)}, \quad\left[M_{\hat{\alpha} \hat{\beta}}, \mathcal{D}_{\hat{\gamma}}^{k}\right]=\varepsilon_{\hat{\gamma}(\hat{\alpha}} \mathcal{D}_{\hat{\beta}}^{k}, \quad\left[M_{\hat{a} \hat{b}}, \mathcal{D}_{\hat{c}}\right]=2 \eta_{\hat{c}[\hat{a}} \mathcal{D}_{\hat{b}]},
$$

where $J^{k l}=\varepsilon^{k i} \varepsilon^{l j} J_{i j}$.

The supergravity gauge group is generated by local transformations of the form

$$
\delta_{\mathcal{K}} \mathcal{D}_{\hat{A}}=\left[\mathcal{K}, \mathcal{D}_{\hat{A}}\right], \quad \mathcal{K}=\xi^{\hat{C}}(z) \mathcal{D}_{\hat{C}}+\frac{1}{2} K^{\hat{c} \hat{d}}(z) M_{\hat{c} \hat{d}}+K^{k l}(z) J_{k l},
$$

with all the gauge parameters obeying natural reality conditions but are otherwise arbitrary. Given a tensor superfield $U(z)$ (with its indices suppressed), its transformation law under the supergravity gauge group is

$$
\delta_{\mathcal{K}} U=\mathcal{K} U
$$

By construction, the covariant derivatives have (anti-)commutation relations of the general form

$$
\left[\mathcal{D}_{\hat{A}}, \mathcal{D}_{\hat{B}}\right\}=T_{\hat{A} \hat{B}} \hat{C} \mathcal{D}_{\hat{C}}+\frac{1}{2} R_{\hat{A} \hat{B}}^{\hat{c} \hat{d}} M_{\hat{c} \hat{d}}+R_{\hat{A} \hat{B}}^{k l} J_{k l},
$$

where $T_{\hat{A} \hat{B}}^{\hat{C}}$ is the torsion, and $R_{\hat{A} \hat{B}} \hat{c} \hat{d}$ and $R_{\hat{A} \hat{B}}^{k l}$ are the $\mathrm{SO}(4,1)$ and $\mathrm{SU}(2)$ curvature tensors, respectively.

To describe conformal supergravity, the covariant derivatives have to obey certain constraints [3]. Upon solving the Bianchi identities for the constraints imposed, it can be shown that the covariant derivatives are characterized by the (anti-)commutation relations:

$$
\begin{aligned}
& \left\{\mathcal{D}_{\hat{\alpha}}^{i}, \mathcal{D}_{\hat{\beta}}^{j}\right\}=-2 \mathrm{i} \varepsilon^{i j} \mathcal{D}_{\hat{\alpha} \hat{\beta}}-\mathrm{i} \varepsilon_{\hat{\alpha} \hat{\beta}} \varepsilon^{i j} X^{\hat{c} \hat{d}} M_{\hat{c} \hat{d}}+\frac{\mathrm{i}}{4} \varepsilon^{i j} \varepsilon^{\hat{a} \hat{b} \hat{c} \hat{d} \hat{e}}\left(\Gamma_{\hat{a}}\right)_{\hat{\alpha} \hat{\beta}} N_{\hat{b} \hat{c}} M_{\hat{d} \hat{e}} \\
& -\frac{\mathrm{i}}{2} \varepsilon^{\hat{a} \hat{b} \hat{c} \hat{d} \hat{e}}\left(\Sigma_{\hat{a} \hat{b}}\right)_{\hat{\alpha} \hat{\beta}} C_{\hat{c}}^{i j} M_{\hat{d} \hat{e}}+4 \mathrm{i} S^{i j} M_{\hat{\alpha} \hat{\beta}}+3 \mathrm{i} \varepsilon_{\hat{\alpha} \hat{\beta}} \varepsilon^{i j} S^{k l} J_{k l} \\
& -\mathrm{i} \varepsilon^{i j} C_{\hat{\alpha} \hat{\beta}}^{k l} J_{k l}-4 \mathrm{i}\left(X_{\hat{\alpha} \hat{\beta}}+N_{\hat{\alpha} \hat{\beta}}\right) J^{i j} \text {, } \\
& {\left[\mathcal{D}_{\hat{a}}, \mathcal{D}_{\hat{\beta}}^{j}\right]=\frac{1}{2}\left(\left(\Gamma_{\hat{a}}\right)_{\hat{\beta}}{ }^{\hat{\gamma}} S^{j}{ }_{k}-X_{\hat{a} \hat{b}}\left(\Gamma^{\hat{b}}\right)_{\hat{\beta}} \hat{\gamma} \delta_{k}^{j}-\frac{1}{4} \varepsilon_{\hat{a} \hat{b} \hat{c} \hat{d} \hat{e}} N^{\hat{d} \hat{e}}\left(\Sigma^{\hat{b} \hat{c}}\right)_{\hat{\beta}} \hat{\gamma} \delta_{k}^{j}+\left(\Sigma_{\hat{a}}{ }^{\hat{b}}\right)_{\hat{\beta}} \hat{\gamma}^{\hat{\gamma}} C_{\hat{b}}^{j} k\right) \mathcal{D}_{\hat{\gamma}}^{k}} \\
& -\frac{\mathrm{i}}{2}\left(\left(\Gamma_{\hat{a}}\right)_{\hat{\beta}}{ }^{\hat{\gamma}} T_{\hat{\gamma}}^{\hat{c} \hat{d} j}+2\left(\Gamma^{[\hat{c}}\right)_{\hat{\beta}}^{\hat{\gamma}} T_{\hat{a}}^{\hat{d}}{ }_{\hat{\gamma}} j\right] M_{\hat{c} \hat{d}} \\
& +\left(3 \Xi_{\hat{a}}^{(k} \varepsilon^{l l) j}-\frac{1}{3} \mathcal{C}_{\hat{a}}^{(k} \varepsilon^{l) j}-\frac{5}{4}\left(\Gamma_{\hat{a}}\right)_{\hat{\beta}} \hat{\gamma}_{\hat{\gamma}}^{(k} \varepsilon^{l) j}+\frac{1}{4}\left(\Gamma_{\hat{a}}\right)_{\hat{\beta}} \hat{\gamma}^{(k} \mathcal{N}_{\hat{\gamma}}^{(k)} \varepsilon^{l) j}\right. \\
& \left.+\frac{1}{8}\left(\Gamma_{\hat{a}}\right)_{\hat{\beta}} \hat{\mathcal{C}}_{\hat{\gamma}}^{j k l}-\frac{11}{24}\left(\Gamma_{\hat{a}}\right)_{\hat{\beta}}^{\hat{\gamma}} \mathcal{C}_{\hat{\gamma}}^{(k} \varepsilon^{l) j}\right) J_{k l}
\end{aligned}
$$

The algebra of covariant derivatives is given in terms of dimension-1 tensor superfields, $S^{i j}$, $X_{\hat{a} \hat{b}}, N_{\hat{a} \hat{b}}$ and $C_{\hat{a}}^{i j}$, and their covariant derivatives. They possess the symmetry properties:

$$
S^{i j}=S^{j i}, \quad X_{\hat{a} \hat{b}}=-X_{\hat{b} \hat{a}}, \quad N_{\hat{a} \hat{b}}=-N_{\hat{b} \hat{a}}, \quad C_{\hat{a}}^{i j}=C_{\hat{a}}^{j i} .
$$

\footnotetext{
${ }^{3}$ The operation of (anti-)symmetrization of $n$ indices is defined to involve a factor $(n !)^{-1}$.
} 
Their reality properties are

$$
\overline{S^{i j}}=S_{i j}, \quad \overline{X_{\hat{a} \hat{b}}}=X_{\hat{a} \hat{b}}, \quad \overline{N_{\hat{a} \hat{b}}}=N_{\hat{a} \hat{b}}, \quad \overline{C_{\hat{a}}^{i j}}=C_{\hat{a} i j} .
$$

The torsion superfields (2.9) enjoy some additional differential constraints that follow from the Bianchi identities. In terms of the irreducible components of $\mathcal{D}_{\hat{\gamma}}^{k} X_{\hat{a} \hat{b}}$ and $\mathcal{D}_{\hat{\gamma}}^{k} C_{\hat{a}}^{i j}$ defined by

$$
\begin{aligned}
\mathcal{D}_{\hat{\gamma}}^{k} X_{\hat{a} \hat{b}} & =W_{\hat{a} \hat{b} \hat{\gamma}}^{k}+2\left(\Gamma_{[\hat{a}}\right)_{\hat{\gamma}}^{\hat{\delta}} \Xi_{\hat{b}] \hat{\delta}}^{k}+\left(\Sigma_{\hat{a} \hat{b}}\right)_{\hat{\gamma}}^{\hat{\delta}} \mathcal{F}_{\hat{\delta}}^{k}, \\
\left(\Gamma^{\hat{a}}\right)_{\hat{\alpha}}^{\hat{\beta}} \Xi_{\hat{a} \hat{\beta}}{ }^{i} & =\left(\Gamma^{\hat{a}}\right)_{\hat{\alpha}}^{\hat{\beta}} W_{\hat{a} \hat{b} \hat{\beta}}{ }^{i}=0, \\
\mathcal{D}_{\hat{\gamma}}^{k} C_{\hat{a}}^{i j} & =\mathcal{C}_{\hat{a} \hat{\gamma}}{ }^{i j k}-\frac{2}{3} \mathcal{C}_{\hat{a} \hat{\gamma}}^{(i} \varepsilon^{j) k}-\frac{1}{2}\left(\Gamma_{\hat{a}}\right)_{\hat{\gamma}}{ }^{\hat{\delta}} \mathcal{C}_{\hat{\delta}}^{i j k}+\frac{1}{3}\left(\Gamma_{\hat{a}}\right)_{\hat{\gamma}}{ }^{\hat{\delta}} \mathcal{C}_{\hat{\delta}}^{(i} \varepsilon^{j) k}, \\
\mathcal{C}_{\hat{a} \hat{\gamma}}{ }^{i j k} & =\mathcal{C}_{\hat{a} \hat{\gamma}}{ }^{(i j k)}, \quad \mathcal{C}_{\hat{\delta}}^{i j k}=\mathcal{C}_{\hat{\delta}}^{(i j k)}, \quad\left(\Gamma^{\hat{a}}\right)_{\hat{\alpha}}^{\hat{\beta}} \mathcal{C}_{\hat{a} \hat{\beta}}{ }^{i j k}=0,
\end{aligned}
$$

the dimension-3/2 Bianchi identities are:

$$
\begin{aligned}
\mathcal{D}_{\hat{\gamma}}^{k} N_{\hat{a} \hat{b}} & =-W_{\hat{a} \hat{b} \hat{\gamma}}^{k}+4\left(\Gamma_{[\hat{a}}\right)_{\hat{\gamma}}^{\hat{\delta}} \Xi_{\hat{b}] \hat{\delta}}^{k}+\left(\Sigma_{\hat{a} \hat{b}}\right) \hat{\gamma}_{\hat{\gamma}} \mathcal{N}_{\hat{\delta}}^{k}, \\
\mathcal{C}_{\hat{a} \hat{\gamma}} i j k & =0 \\
\mathcal{D}_{\hat{\gamma}}^{k} S^{i j} & =-\frac{1}{4} \mathcal{C}_{\hat{\gamma}}^{i j k}+\frac{5}{12} \mathcal{C}_{\hat{\gamma}}^{(i} \varepsilon^{j) k}+\frac{1}{2}\left(3 \mathcal{F}_{\hat{\gamma}}^{(i}+\mathcal{N}_{\hat{\gamma}}^{(i}\right) \varepsilon^{j) k} .
\end{aligned}
$$

The tensor $T_{\hat{a} \hat{b} k}^{\hat{\gamma}}$ in (2.8b) is the dimension-3/2 torsion. Its explicit form is

$$
T_{\hat{a} \hat{b} \hat{\gamma}}^{k}=\frac{\mathrm{i}}{2} \mathcal{D}_{\hat{\gamma}}^{k} X_{\hat{a} \hat{b}}-\frac{\mathrm{i}}{6}\left(\Gamma_{[\hat{a}}\right)_{\hat{\gamma}}^{\hat{\delta}} \mathcal{C}_{\hat{b}] \hat{\delta}}^{k}+\frac{\mathrm{i}}{4}\left(\Sigma_{\hat{a} \hat{b}}\right) \hat{\gamma} \hat{\mathcal{C}}_{\hat{\delta}}^{\hat{\delta}}
$$

The above superspace geometry describes conformal supergravity due to the fact that the algebra of covariant derivatives is invariant under infinitesimal super-Weyl transformations of the form

$$
\begin{aligned}
\delta_{\sigma} \mathcal{D}_{\hat{\alpha}}^{i} & =\frac{1}{2} \sigma \mathcal{D}_{\hat{\alpha}}^{i}+2\left(\mathcal{D}^{\hat{\gamma} i} \sigma\right) M_{\hat{\gamma} \hat{\alpha}}-3\left(\mathcal{D}_{\hat{\alpha} k} \sigma\right) J^{k i} \\
\delta_{\sigma} \mathcal{D}_{\hat{a}} & =\sigma \mathcal{D}_{\hat{a}}+\frac{i}{2}\left(\Gamma_{\hat{a}}\right)^{\hat{\gamma} \hat{\delta}}\left(\mathcal{D}_{\hat{\gamma}}^{k} \sigma\right) \mathcal{D}_{\hat{\delta} k}-\left(\mathcal{D}^{\hat{b}} \sigma\right) M_{\hat{a} \hat{b}}+\frac{\mathrm{i}}{8}\left(\Gamma_{\hat{a}}\right)^{\hat{\gamma} \hat{\delta}}\left(\mathcal{D}_{\hat{\gamma}}^{(k} \mathcal{D}_{\hat{\delta}}^{l)} \sigma\right) J_{k l},
\end{aligned}
$$

provided the components of the torsion transform as follows:

$$
\begin{aligned}
\delta_{\sigma} S^{i j} & =\sigma S^{i j}+\frac{\mathrm{i}}{4} \mathcal{D}^{\hat{\alpha}(i} \mathcal{D}_{\hat{\alpha}}^{j)} \sigma \\
\delta_{\sigma} C_{\hat{a}}^{i j} & =\sigma C_{\hat{a}}^{i j}+\frac{\mathrm{i}}{2}\left(\Gamma_{\hat{a}}\right)^{\hat{\gamma} \hat{\delta}} \mathcal{D}_{\hat{\gamma}}^{(i} \mathcal{D}_{\hat{\delta}}^{j)} \sigma \\
\delta_{\sigma} X_{\hat{a} \hat{b}} & =\sigma X_{\hat{a} \hat{b}}-\frac{\mathrm{i}}{4}\left(\Sigma_{\hat{a} \hat{b}}\right)^{\hat{\alpha} \hat{\beta}} \mathcal{D}_{\hat{\alpha}}^{k} \mathcal{D}_{\hat{\beta} k} \sigma \\
\delta_{\sigma} N_{\hat{a} \hat{b}} & =\sigma N_{\hat{a} \hat{b}}-\frac{\mathrm{i}}{2}\left(\Sigma_{\hat{a} \hat{b}}\right)^{\hat{\alpha} \hat{\beta}} \mathcal{D}_{\hat{\alpha}}^{k} \mathcal{D}_{\hat{\beta} k} \sigma
\end{aligned}
$$

with the parameter $\sigma(z)$ being an arbitrary real scalar superfield. ${ }^{4}$ It follows that the tensor

$$
W_{\hat{a} \hat{b}}:=X_{\hat{a} \hat{b}}-\frac{1}{2} N_{\hat{a} \hat{b}}
$$

\footnotetext{
${ }^{4}$ The finite form for the super-Weyl transformations is given in [49]. As compared with [3, 49], we have rescaled the super-Weyl parameter $\sigma \rightarrow \frac{1}{2} \sigma$.
} 
transforms homogeneously,

$$
\delta_{\sigma} W_{\hat{a} \hat{b}}=\sigma W_{\hat{a} \hat{b}},
$$

and hence is a superspace generalization of the Weyl tensor.

In complete analogy with $\mathcal{N}=2$ supergravity in four dimensions (see, e.g., [50] for a review), $5 \mathrm{D} \mathcal{N}=1$ Poincaré or anti-de Sitter supergravity theories are obtained by coupling the Weyl multiplet with two off-shell conformal compensators, one of which is (almost) invariably a vector multiplet. Conceptually, this approach is a natural extension of the Weyl-invariant formulation for gravity reviewed in appendix A.

\section{3 (Conformal) isometries}

Consider some background superspace $\mathcal{M}^{5 \mid 8}$ such that its geometry is of the type described in the previous section. In order to formulate rigid superconformal or rigid supersymmetric field theories on $\mathcal{M}^{5 \mid 8}$, one has to determine all (conformal) isometries of this superspace. This can be done similarly to the case of $4 \mathrm{D} \mathcal{N}=1$ supergravity described in detail in [19] and elaborated in [45]. A similar analysis in the case of $3 \mathrm{D} \mathcal{N}=2$ supergravity has recently been carried out in [46].

\subsection{Conformal isometries}

Let $\xi=\xi^{\hat{A}} E_{\hat{A}}=\xi^{\hat{a}} E_{\hat{a}}+\xi_{i}^{\hat{\alpha}} E_{\hat{\alpha}}^{i}$ be a real supervector field on $\mathcal{M}^{5 \mid 8}$. It is called conformal Killing if one can associate with $\xi$ a supergravity gauge transformation (2.5) and an infinitesimal super-Weyl transformation (2.14) such that their combined action

$$
\delta:=\delta_{\mathcal{K}}+\delta_{\sigma}
$$

does not change the covariant derivatives,

$$
\delta \mathcal{D}_{\hat{A}}=0
$$

These conditions, which appeared for the first time in [3], clearly imply that all the torsion and curvature tensors are invariant under the transformation $\delta$. One may see that it suffices to demand only the spinor condition $\delta \mathcal{D}_{\hat{\alpha}}^{i}=0$ in order for (3.2) to hold. A short calculation gives

$$
\begin{aligned}
\delta \mathcal{D}_{\hat{\alpha}}^{i}= & \left(\xi^{\hat{C}} T_{\hat{C} \hat{\alpha} j}^{i \hat{\beta}}-\mathcal{D}_{\hat{\alpha}}^{i} \xi_{j}^{\hat{\beta}}+K_{\hat{\alpha}}^{\hat{\beta}} \delta_{j}^{i}+K^{i}{ }_{j} \delta_{\hat{\alpha}}^{\hat{\beta}}+\frac{1}{2} \sigma \delta_{\hat{\alpha}}^{\hat{\beta}} \delta_{j}^{i}\right) \mathcal{D}_{\hat{\beta}}^{j} \\
& +\left(\xi^{\hat{C}} T_{\hat{C} \hat{\alpha}}^{i \hat{b}}-\mathcal{D}_{\hat{\alpha}}^{i} \xi^{\hat{b}}\right) \mathcal{D}_{\hat{b}} \\
& +\left(\xi^{\hat{D}} R_{\hat{D} \hat{\alpha} \hat{\beta} \hat{\gamma}}^{i}-\mathcal{D}_{\hat{\alpha}}^{i} K_{\hat{\beta} \hat{\gamma}}-2 \varepsilon_{\hat{\alpha}(\hat{\beta}} \mathcal{D}_{\hat{\gamma})}^{i} \sigma\right) M^{\hat{\beta} \hat{\gamma}} \\
& +\left(\xi^{\hat{D}} R_{\hat{D} \hat{\alpha}}^{i j k}-\mathcal{D}_{\hat{\alpha}}^{i} K^{j k}+3 \varepsilon^{i(j} \mathcal{D}_{\hat{\alpha}}^{k)} \sigma\right) J_{j k} .
\end{aligned}
$$

The right-hand side of (3.3) is a combination of the four linearly independent operators $\mathcal{D}_{\hat{\beta}}^{j}, \mathcal{D}_{\hat{b}}, M^{\hat{\beta} \hat{\gamma}}$ and $J_{j k}$. Requiring $\delta \mathcal{D}_{\hat{\alpha}}^{i}=0$ leads to four different equations. Making use of 
the explicit form of the torsion, the equations associated with the operators $\mathcal{D}_{\hat{\beta}}^{j}$ and $\mathcal{D}_{\hat{b}}$ in the right-hand side of (3.3) may be written as

$$
\begin{aligned}
\mathcal{D}_{\hat{\alpha}}^{i} \xi_{\hat{\beta}}^{j}= & \frac{1}{2} \xi^{\hat{a}}\left(\left(\Gamma_{\hat{a}}\right)_{\hat{\alpha} \hat{\beta}} S^{i j}+X_{\hat{a} \hat{b}}\left(\Gamma^{\hat{b}}\right)_{\hat{\alpha} \hat{\beta}} \varepsilon^{i j}+\frac{1}{4} \varepsilon_{\hat{a} \hat{b} \hat{c} \hat{d} \hat{e}} N^{\hat{b} \hat{c}}\left(\Sigma^{\hat{d} \hat{e}}\right)_{\hat{\alpha} \hat{\beta}} \varepsilon^{i j}+\left(\sum_{\hat{a}}\right)_{\hat{\alpha} \hat{\beta}} C_{\hat{b}}^{i j}\right) \\
& -K_{\hat{\alpha} \hat{\beta}} \varepsilon^{i j}-K^{i j} \varepsilon_{\hat{\alpha} \hat{\beta}}+\frac{1}{2} \sigma \varepsilon_{\hat{\alpha} \hat{\beta}} \varepsilon^{i j} \\
\mathcal{D}_{\hat{\alpha}}^{i} \xi_{\hat{b}}= & 2 \mathrm{i}\left(\Gamma_{\hat{b}}\right)_{\hat{\alpha}}^{\hat{\delta}} \xi_{\hat{\delta}}^{i} .
\end{aligned}
$$

After introducing $\xi_{\hat{\alpha} \hat{\beta}}=\left(\Gamma^{\hat{a}}\right)_{\hat{\alpha} \hat{\beta}} \xi_{\hat{a}}$, equation (3.4b) is equivalent to

$$
\mathcal{D}_{\hat{\alpha}}^{i} \xi_{\hat{\beta} \hat{\gamma}}=-8 \mathrm{i}\left(\xi_{[\hat{\beta}}^{i} \varepsilon_{\hat{\gamma}] \hat{\alpha}}+\frac{1}{4} \xi_{\hat{\alpha}}^{i} \varepsilon_{\hat{\beta} \hat{\gamma}}\right) .
$$

The relations (3.4) imply that the parameters $\xi_{i}^{\hat{\alpha}}, K_{\hat{\alpha} \hat{\beta}}, K^{i j}$ and $\sigma$ are uniquely expressed in terms of $\xi^{\hat{a}}$ and its covariant derivatives as follows:

$$
\begin{aligned}
\xi_{\hat{\alpha}}^{i} & =\frac{\mathrm{i}}{10}\left(\Gamma^{\hat{a}}\right)_{\hat{\alpha}}^{\hat{\beta}} \mathcal{D}_{\hat{\beta}}^{i} \xi_{\hat{a}}, \\
K_{\hat{\alpha} \hat{\beta}} & =\frac{1}{2} \mathcal{D}_{(\hat{\alpha}}^{k} \xi_{k \hat{\beta})}+\frac{1}{8} \xi^{\hat{a}} \varepsilon_{\hat{a} \hat{b} \hat{c} \hat{d} \hat{e}} N^{\hat{b} \hat{c}}\left(\Sigma^{\hat{d} \hat{e}}\right)_{\hat{\alpha} \hat{\beta}} \\
K^{i j} & =\frac{1}{4} \mathcal{D}_{\hat{\gamma}}^{(i} \xi^{j) \hat{\gamma}}=\frac{\mathrm{i}}{40}\left(\Gamma^{\hat{c}}\right)^{\hat{\alpha} \hat{\beta}} \mathcal{D}_{\hat{\alpha}}^{(i} \mathcal{D}_{\hat{\beta}}^{j} \xi_{\hat{c}} \\
\sigma & =\frac{1}{4} \mathcal{D}_{\hat{\alpha}}^{i} \xi_{i}^{\hat{\alpha}} .
\end{aligned}
$$

Since all the parameters in $\mathcal{K}$ and the super-Weyl parameter $\sigma$ are functions of $\xi$, we may use the notation $\mathcal{K}=\mathcal{K}[\xi]$ and $\sigma=\sigma[\xi]$. It is important to note that equation (3.4b) implies a fundamental constraint on $\xi^{\hat{a}}$,

$$
\left(\delta_{\hat{\alpha}}^{\hat{\beta}} \delta_{\hat{a}}^{\hat{b}}+\frac{1}{5}\left(\Gamma_{\hat{a}} \Gamma^{\hat{b}}\right)_{\hat{\alpha}}^{\hat{\beta}}\right) \mathcal{D}_{\hat{\beta}}^{i} \xi_{\hat{b}}=\frac{4}{5}\left(\delta_{\hat{\alpha}}^{\hat{\beta}} \delta_{\hat{a}}^{\hat{b}}-\frac{1}{2}\left(\Sigma_{\hat{a}}^{\hat{b}}\right)_{\hat{\alpha}}^{\hat{\beta}}\right) \mathcal{D}_{\hat{\beta}}^{i} \xi_{\hat{b}}=0
$$

This equation means that the gamma-traceless component of the spinor-vector $\mathcal{D}_{\hat{\alpha}}^{i} \xi_{\hat{b}}$ is zero. Eqs. (3.6) and (3.7) imply the conformal Killing equation

$$
\mathcal{D}_{(\hat{a}} \xi_{\hat{b})}=\frac{1}{5} \eta_{\hat{a} \hat{b}} \mathcal{D}_{\hat{c}} \xi^{\hat{c}}
$$

Other consequences of (3.6) and (3.7) are

$$
\begin{aligned}
\sigma & =\frac{1}{5} \mathcal{D}_{\hat{c}} \xi^{\hat{c}}, \\
K_{\hat{a} \hat{b}} & =\mathcal{D}_{[\hat{a}} \xi_{\hat{b}]} \quad \Longleftrightarrow \quad K_{\hat{\alpha} \hat{\beta}}=\frac{1}{2}\left(\Sigma^{\hat{a} \hat{b}}\right)_{\hat{\alpha} \hat{\beta}} \mathcal{D}_{\hat{a}} \xi_{\hat{b}} .
\end{aligned}
$$

If eq. (3.7) holds and the conditions (3.6) are adopted, it can be proved that equation (3.2) is identically satisfied. Therefore, (3.7) is the fundamental equation containing all the information about the conformal Killing supervector fields. This means that the conformal Killing supervector field can alternatively be defined as a real supervector field,

$$
\xi=\xi^{\hat{A}} E_{\hat{A}}, \quad \xi^{\hat{A}} \equiv\left(\xi^{\hat{a}}, \xi_{i}^{\hat{\alpha}}\right)=\left(\xi^{\hat{a}}, \frac{\mathrm{i}}{10} \mathcal{D}_{\hat{\beta} i} \xi^{\hat{\alpha} \hat{\beta}}\right)
$$

obeying the master equation (3.7). 
If $\xi_{1}$ and $\xi_{2}$ are two conformal Killing supervector fields, their Lie bracket $\left[\xi_{1}, \xi_{2}\right]$ is a conformal Killing supervector field. It is obvious that for any real $c$-numbers $r_{1}$ and $r_{2}$, the linear combination $r_{1} \xi_{1}+r_{2} \xi_{2}$ is a conformal Killing supervector field. Thus the set of all conformal Killing supervector fields is a super Lie algebra. The conformal Killing supervector fields of $\mathcal{M}^{5 \mid 8}$ generate symmetries of a superconformal field theory on this superspace.

We have not yet analysed the equations associated with the generators $M^{\hat{\beta} \hat{\gamma}}$ and $J_{j k}$ in the right-hand side of (3.3). They are

$$
\begin{aligned}
& \mathcal{D}_{\hat{\alpha}}^{i} K_{\hat{\beta} \hat{\gamma}}=\xi^{\hat{D}} R_{\hat{D} \hat{\alpha} \hat{\beta} \hat{\gamma}}^{i}-2 \varepsilon_{\hat{\alpha}(\hat{\beta}} \mathcal{D}_{\hat{\gamma})}^{i} \sigma \\
& \mathcal{D}_{\hat{\alpha}}^{i} K^{j k}=\xi^{\hat{D}} R_{\hat{D} \hat{\alpha}}^{i j k}+3 \varepsilon^{i(j} \mathcal{D}_{\hat{\alpha}}^{k)} \sigma .
\end{aligned}
$$

The relations (3.4) tell us that any spinor covariant derivative of $\xi^{\hat{B}}$ can be represented as a linear combination of the parameters $\Upsilon=\left(\xi^{\hat{B}}, K^{\hat{\beta} \hat{\gamma}}, K^{j k}, \sigma\right)$. The relations (3.11) also tell us that $\mathcal{D}_{\hat{\alpha}}^{i} \Upsilon$ can be represented as a linear combination of $\Upsilon$ and $\mathcal{D}_{\hat{\gamma}}^{k} \sigma$. It turns out that $\mathcal{D}_{\hat{a}} \Upsilon$ may be represented as a linear combination of $\Upsilon$ and $\mathcal{D}_{\hat{C}} \sigma$. To prove this claim, let us look at the conditions of invariance of the dimension- 1 torsion superfields, $\delta S^{i j}=0$, $\delta C_{\hat{a}}^{i j}=0, \delta X^{\hat{a} \hat{b}}=0$ and $\delta N^{\hat{a} \hat{b}}=0$. These conditions ${ }^{5}$ are:

$$
\begin{aligned}
& \mathcal{D}^{\hat{\alpha}(i} \mathcal{D}_{\hat{\alpha}}^{j)} \sigma=4 \mathrm{i} \xi^{\hat{C}} \mathcal{D}_{\hat{C}} S^{i j}+8 \mathrm{i} K^{(i}{ }_{k} S^{j) k}+4 \mathrm{i} \sigma S^{i j}, \\
& \left(\Gamma_{\hat{a}}\right)^{\hat{\gamma} \hat{\delta}} \mathcal{D}_{\hat{\gamma}}^{(i} \mathcal{D}_{\hat{\delta}}^{j)} \sigma=2 \mathrm{i} \xi^{\hat{C}} \mathcal{D}_{\hat{C}} C_{\hat{a}}{ }^{i j}+2 \mathrm{i} K_{\hat{a}}{ }^{\hat{b}} C_{\hat{b}}{ }^{i j}+4 \mathrm{i} K^{(i}{ }_{k} C_{\hat{a}}{ }^{j) k}+\sigma C_{\hat{a}}{ }^{i j}, \\
& \left(\Sigma_{\hat{a} \hat{b}}\right)^{\hat{\alpha} \hat{\beta}} \mathcal{D}_{\hat{\alpha}}^{k} \mathcal{D}_{\hat{\beta} k} \sigma=-4 \mathrm{i} \xi^{\hat{C}} \mathcal{D}_{\hat{C}} X_{\hat{a} \hat{b}}+8 \mathrm{i} K_{[\hat{a}}^{\hat{c}} X_{\hat{b}] \hat{c}}-4 \mathrm{i} \sigma X_{\hat{a} \hat{b}}, \\
& \left(\Sigma_{\hat{a} \hat{b}}\right)^{\hat{\alpha} \hat{\beta}} \mathcal{D}_{\hat{\alpha}}^{k} \mathcal{D}_{\hat{\beta} k} \sigma=-2 \mathrm{i} \xi^{\hat{C}} \mathcal{D}_{\hat{C}} N_{\hat{a} \hat{b}}+4 \mathrm{i} K_{[\hat{a}}^{\hat{c}} N_{\hat{b}] \hat{c}}-2 \mathrm{i} \sigma N_{\hat{a} \hat{b}} .
\end{aligned}
$$

These identities tell us that two spinor derivatives of $\sigma$ may be represented as a linear combination of $\Upsilon$ and $\mathcal{D}_{\hat{C}} \sigma$. This confirms the above claim. Furthermore, it is not hard to deduce from the above identities that $\mathcal{D}_{\hat{\alpha}}^{i} \mathcal{D}_{\hat{B}} \sigma$ may be represented as a linear combination of $\Upsilon$ and $\mathcal{D}_{\hat{C}} \sigma$. As a result, applying any number of covariant derivatives to $\Upsilon$ gives a linear combination of $\Upsilon$ and $\mathcal{D}_{\hat{C}} \sigma$. We conclude that the super Lie algebra of the conformal Killing vector fields on $\mathcal{M}^{5 \mid 8}$ is finite dimensional. The number of its even and odd generators cannot exceed those in the 5D superconformal algebra $\mathfrak{f}(4)$.

To study supersymmetry transformations at the component level, it is useful to spell out one of the implications of (3.2) with $\hat{A}=\hat{a}$. Specifically, we consider the equation $\delta \mathcal{D}_{\hat{a}}=0$ and read off the part proportional to a linear combination of the spinor covariant derivatives $\mathcal{D}_{\hat{\gamma}}^{k}$. The result is

$$
\begin{aligned}
0= & \mathcal{D}_{\hat{a}} \xi_{k}^{\hat{\gamma}}-\frac{1}{2}\left(S_{k}^{l}\left(\Gamma_{\hat{a}}\right)_{\hat{\delta}}^{\hat{\delta}}-X_{\hat{a} \hat{b}}\left(\Gamma^{\hat{b}}\right)^{\hat{\gamma}} \delta_{k}^{l}+\frac{1}{4} \delta_{k}^{l} \varepsilon_{\hat{a} \hat{b} \hat{c} \hat{d} \hat{e}} N^{\hat{d} \hat{e}}\left(\sum^{\hat{b} \hat{c}}\right)^{\hat{\gamma}}{ }_{\hat{\delta}}-\left(\sum_{\hat{a}}{ }^{\hat{b}}\right)^{\hat{\gamma}} C_{\hat{\delta} k}{ }^{l}\right) \xi_{l}^{\hat{\delta}} \\
& +\xi^{\hat{b}} T_{\hat{a} \hat{b} k}^{\hat{\gamma}}-\frac{\mathrm{i}}{2}\left(\Gamma_{\hat{a}}\right)^{\hat{\gamma} \hat{\delta}} \mathcal{D}_{\hat{\delta} k} \sigma .
\end{aligned}
$$

\footnotetext{
${ }^{5}$ The conditions (3.12) are not new constraints. They are satisfied identically provided eq. (3.2) holds.
} We should point out that eqs. (3.12c) and (3.12d) imply the invariance condition of the super-Weyl tensor, which is $\xi^{\hat{C}} \mathcal{D}_{\hat{C}} W_{\hat{a} \hat{b}}-2 K_{[\hat{a}}^{\hat{c}} W_{\hat{b}] \hat{c}}+\sigma W_{\hat{a} \hat{b}}=0$. 
It should be mentioned that (3.13) is not a new constraint. It is satisfied identically provided the spinor condition $\delta \mathcal{D}_{\hat{\alpha}}^{i}=0$ holds.

\subsection{Conformally related superspaces}

A superspace $\widetilde{\mathcal{M}}^{5 \mid 8}$ is said to be conformally related to $\mathcal{M}^{5 / 8}$ if the covariant derivatives $\widetilde{\mathcal{D}}_{\hat{A}}$ of $\widetilde{\mathcal{M}}^{5 \mid 8}$ are obtained from $\mathcal{D}_{\hat{A}}$ by a finite super-Weyl transformation [49],

$$
\begin{aligned}
\widetilde{\mathcal{D}}_{\hat{\alpha}}^{i}= & \mathrm{e}^{\frac{1}{2} \rho}\left(\mathcal{D}_{\hat{\alpha}}^{i}+2\left(\mathcal{D}^{\hat{\beta} i} \rho\right) M_{\hat{\alpha} \hat{\beta}}-3\left(\mathcal{D}_{\hat{\alpha} j} \rho\right) J^{i j}\right), \\
\widetilde{\mathcal{D}}_{\hat{a}}= & \mathrm{e}^{\rho}\left(\mathcal{D}_{\hat{a}}+\frac{\mathrm{i}}{2}\left(\Gamma_{\hat{a}}\right)^{\hat{\gamma} \hat{\delta}}\left(\mathcal{D}_{\hat{\gamma}}^{k} \rho\right) \mathcal{D}_{\hat{\delta} k}-\left(\mathcal{D}^{\hat{b}} \rho\right) M_{\hat{a} \hat{b}}+\frac{\mathrm{i}}{8}\left(\Gamma_{\hat{a}}\right)^{\hat{\gamma} \hat{\delta}}\left(\mathcal{D}_{\hat{\gamma}}^{k} \mathcal{D}_{\hat{\delta}}^{l} \rho\right) J_{k l}\right. \\
& \left.+\frac{\mathrm{i}}{8} \varepsilon_{\hat{a} \hat{b} \hat{c} \hat{d} \hat{e}}\left(\Sigma^{\hat{b} \hat{c}}\right)_{\hat{\gamma} \hat{\delta}}\left(\mathcal{D}^{\hat{\gamma} k} \rho\right)\left(\mathcal{D}_{k}^{\hat{\delta}} \rho\right) M^{\hat{d} \hat{e}}+\frac{5 \mathrm{i}}{8}\left(\Gamma_{\hat{a}}\right)^{\hat{\gamma} \hat{\delta}}\left(\mathcal{D}_{\hat{\gamma}}^{k} \rho\right)\left(\mathcal{D}_{\hat{\delta}}^{l} \rho\right) J_{k l}\right),
\end{aligned}
$$

for some super-Weyl parameter $\rho$. The two superspaces $\widetilde{\mathcal{M}}{ }^{5 \mid 8}$ and $\mathcal{M}^{5 \mid 8}$ prove to have the same conformal Killing supervector fields. Given such a vector field $\xi=\xi^{\hat{A}} E_{\hat{A}}=\widetilde{\xi}^{\hat{A}} \widetilde{E}_{\hat{A}}$, it may be shown that

$$
\begin{aligned}
\mathcal{K}[\widetilde{\xi}] & :=\widetilde{\xi}^{\hat{B}} \widetilde{\mathcal{D}}_{\hat{B}}+\frac{1}{2} K^{\hat{b} \hat{c}}[\widetilde{\xi}] M_{\hat{b} \hat{c}}+K^{k l}[\widetilde{\xi}] J_{k l}=\mathcal{K}[\xi] \\
\sigma[\widetilde{\xi}] & =\sigma[\xi]-\xi \rho .
\end{aligned}
$$

This is similar to the $4 \mathrm{D}$ and $3 \mathrm{D}$ analyses in [45] and [46], respectively.

\subsection{Isometries}

In order to describe $\mathcal{N}=1$ Poincaré or anti-de Sitter supergravity theories, the Weyl multiplet has to be coupled with two off-shell conformal compensators that will be symbolically denoted $\Xi$. In general, both compensators are Lorentz scalars and have non-zero super-Weyl weights $w_{\Xi} \neq 0$,

$$
\delta_{\sigma} \Xi=w_{\Xi} \sigma \Xi
$$

They may transform in nontrivial representations of the SU(2) group, which we do not specify at the moment. The compensators are required to be nowhere vanishing in the sense that the SU(2) scalars $|\Xi|^{2}$ should be strictly positive. Different off-shell supergravity theories correspond to different choices of $\Xi$.

The off-shell supergravity multiplet is completely described in terms of the following data: (i) the superspace geometry described in section 2; and (ii) the conformal compensators $\Xi$. Given a supergravity background, its isometries should preserve both of these inputs. This leads us to the concept of Killing supervector fields.

A conformal Killing supervector field $\xi=\xi^{\hat{A}} E_{\hat{A}}$ on $\mathcal{M}^{5 \mid 8}$ is said to be Killing if the following conditions hold:

$$
\begin{aligned}
{\left[\xi^{\hat{B}} \mathcal{D}_{\hat{B}}+\frac{1}{2} K^{\hat{b} \hat{c}}[\xi] M_{\hat{b} \hat{c}}+K^{k l}[\xi] J_{k l}, \mathcal{D}_{\hat{A}}\right]+\delta_{\sigma[\xi]} \mathcal{D}_{\hat{A}} } & =0 \\
\left(\xi^{\hat{B}} \mathcal{D}_{\hat{B}}+K^{k l}[\xi] J_{k l}+w_{\Xi} \sigma[\xi]\right) \Xi & =0 .
\end{aligned}
$$


Here the parameters $K^{\hat{b} \hat{c}}[\xi], K^{k l}[\xi]$ and $\sigma[\xi]$ are defined as in (3.6). The set of all Killing supervector fields on $\mathcal{M}^{5 \mid 8}$ is a super Lie algebra. The Killing supervector fields of $\mathcal{M}^{5 \mid 8}$ generate the spacetime (super)symmetries of all rigid supersymmetric field theories on this superspace.

The Killing equations (3.17) are super-Weyl invariant in the following sense. Consider a supergravity background $\left(\widetilde{\mathcal{D}}_{\hat{A}}, \widetilde{\Xi}\right)$ that is conformal to $\left(\mathcal{D}_{\hat{A}}, \Xi\right)$, where $\widetilde{\mathcal{D}}_{\hat{A}}$ is related to $\mathcal{D}_{\hat{A}}$ according to $(3.14)$ and $\widetilde{\Xi}$ is

$$
\widetilde{\Xi}=\mathrm{e}^{w_{\Xi} \sigma} \Xi
$$

Then the equations (3.17) have the same form once rewritten in terms of $\left(\widetilde{\mathcal{D}}_{\hat{A}}, \widetilde{\Xi}\right)$.

Using the compensators $\Xi$ we can always construct a Lorentz and $\mathrm{SU}(2)$ scalar superfield $\Phi=\Phi(\Xi)$, which is an algebraic function of $\Xi$, nowhere vanishing, and has a nonzero super-Weyl weight $w_{\Phi}$,

$$
\delta_{\sigma} \Phi=w_{\Phi} \sigma \Phi
$$

We have shown that the Killing equations (3.17) are super-Weyl invariant. Super-Weyl invariance may be used to impose the gauge

$$
\Phi=1
$$

Then the equation

$$
\left(\xi^{\hat{B}} \mathcal{D}_{\hat{B}}+K^{k l}[\xi] J_{k l}+w_{\Phi} \sigma[\xi]\right) \Phi=0,
$$

which follows from the Killing equations (3.17b), becomes

$$
\sigma[\xi]=0
$$

The above consideration is analogous to that given in appendix A for the (conformal) isometries of a curved spacetime. The only difference is that a single scalar compensator is used in the case of gravity, while two compensators are needed in order to realize Poincaré or anti-de Sitter supergravities.

\section{Supersymmetric backgrounds: general formalism}

Our analysis will be restricted to curved backgrounds without covariant fermionic fields that is,

$$
\mathcal{D}_{\hat{\alpha}}^{i} S^{k l}\left|=0, \quad \mathcal{D}_{\hat{\alpha}}^{i} C_{\hat{a}}^{k l}\right|=0, \quad \mathcal{D}_{\hat{\alpha}}^{i} X_{\hat{a} \hat{b}}\left|=0, \quad \mathcal{D}_{\hat{\alpha}}^{i} N_{\hat{a} \hat{b}}\right|=0
$$

Here the bar-projection is defined as usual:

$$
U|:=U(x, \theta)|_{\theta=0},
$$


for any superfield $U(z)=U(x, \theta)$. The bar-projection of the superspace covariant derivatives is defined similarly:

$$
\mathcal{D}_{\hat{A}}\left|=E_{\hat{A}}^{\hat{M}}\right| \partial_{\hat{M}}+\frac{1}{2} \Omega_{\hat{A}}^{\hat{b} \hat{c}}\left|M_{\hat{b} \hat{c}}+\Phi_{\hat{A}}^{k l}\right| J_{k l} .
$$

The coordinates $x^{\hat{m}}$ parametrize a curved spacetime $\mathcal{M}^{5}$, the bosonic body of the superspace $\mathcal{M}^{5 \mid 8}$.

The conditions (4.1) mean that the gravitini can completely be gauged away such that the projection of the vector covariant derivatives is

$$
\mathcal{D}_{\hat{a}} \mid=\mathbf{D}_{\hat{a}} \quad \Longleftrightarrow \quad \psi_{\hat{m}_{i}^{\hat{\alpha}}}^{\hat{\alpha}}=0,
$$

where

$$
\mathbf{D}_{\hat{a}}=e_{\hat{a}}+\frac{1}{2} \omega_{\hat{a}}^{\hat{b} \hat{c}} M_{\hat{b} \hat{c}}+\phi_{\hat{a}}^{k l} J_{k l}, \quad e_{\hat{a}}:=e_{\hat{a}}^{\hat{m}} \partial_{\hat{m}}
$$

is a spacetime covariant derivative with Lorentz and $\mathrm{SU}(2)$ connections. In what follows, we always assume that the gravitini have been gauged away. The bosonic covariant derivatives obey commutation relations of the form

$$
\left[\mathbf{D}_{\hat{a}}, \mathbf{D}_{\hat{b}}\right]=\frac{1}{2} \mathcal{R}_{\hat{a} \hat{b}}^{\hat{c} \hat{d}} M_{\hat{c} \hat{d}}+\mathcal{R}_{\hat{a} \hat{b}}^{k l} J_{k l}
$$

where the spacetime curvature tensor $\mathcal{R}_{\hat{a} \hat{b}} \hat{c} \hat{d}$ and the $\mathrm{SU}(2)$ field strength $\mathcal{R}_{\hat{a} \hat{b}} k l$ are related to the superspace ones as

$$
\mathcal{R}_{\hat{a} \hat{b}}^{\hat{c} \hat{d}}=R_{\hat{a} \hat{b}}^{\hat{c} \hat{d}}\left|, \quad \mathcal{R}_{\hat{a} \hat{b}}^{k l}=R_{\hat{a} \hat{b}}^{k l}\right| .
$$

We introduce tensor fields associated with the superspace dimension- 1 torsion tensors:

$$
s^{k l}:=S^{k l}\left|, \quad c_{\hat{a}}^{k l}:=C_{\hat{a}}^{k l}\right|, \quad x_{\hat{a} \hat{b}}:=X_{\hat{a} \hat{b}}\left|, \quad n_{\hat{a} \hat{b}}:=N_{\hat{a} \hat{b}}\right| .
$$

Bar-projecting the super-Weyl tensor gives

$$
w_{\hat{a} \hat{b}}:=W_{\hat{a} \hat{b}} \mid=\frac{1}{2}\left(2 x_{\hat{a} \hat{b}}-n_{\hat{a} \hat{b}}\right) .
$$

\subsection{Conformal Killing spinors}

In this subsection we wish to look for those curved superspace backgrounds which admit at least one conformal supersymmetry. Such a superspace possesses a conformal Killing supervector field $\xi^{\hat{A}}$ with the property

$$
\xi^{\hat{a}}\left|=0, \quad \epsilon_{i}^{\hat{\alpha}}:=\xi_{i}^{\hat{\alpha}}\right| \neq 0 .
$$

All other bosonic parameters will also be assumed to vanish, $K^{\hat{a} \hat{b}}\left|=0, K^{i j}\right|=0$ and $\sigma \mid=0$. The spinor parameter $\epsilon_{i}^{\hat{\alpha}}$ generates a $Q$-supersymmetry transformation, while the $S$-supersymmetry transformations are generated by

$$
\eta_{\hat{\alpha}}^{i}:=\mathcal{D}_{\hat{\alpha}}^{i} \sigma \mid .
$$


With the previous assumptions at hand, bar-projecting the equation (3.13) gives ${ }^{6}$

$$
\mathbf{D}_{\hat{a}} \epsilon^{k}-\frac{1}{2}\left[s^{k}{ }_{l} \Gamma_{\hat{a}}+\delta_{l}^{k} x_{\hat{a} \hat{b}} \Gamma^{\hat{b}}-\frac{1}{4} \delta_{l}^{k} \varepsilon_{\hat{a} \hat{b} \hat{c} \hat{d} \hat{e}} n^{\hat{b} \hat{c}} \sum^{\hat{d} \hat{e}}-c^{\hat{b} k}{ }_{l} \Sigma_{\hat{a} \hat{b}}\right] \epsilon^{l}-\frac{\mathrm{i}}{2} \Gamma_{\hat{a}} \eta^{k}=0,
$$

which implies

$$
5 \eta^{i}=2 \mathrm{i} \Gamma^{\hat{a}} \mathbf{D}_{\hat{a}} \epsilon^{i}+\mathrm{i}\left[2 c_{\hat{a}}{ }^{i}{ }_{j} \Gamma^{\hat{a}}+5 s^{i}{ }_{j} \mathbb{1}+\delta^{i}{ }_{j}\left(4 x_{\hat{a} \hat{b}}+3 n_{\hat{a} \hat{b}}\right) \Sigma^{\hat{a} \hat{b}}\right] \epsilon^{j} .
$$

The spinor equation (4.12) becomes

$$
\mathbf{D}_{\hat{a}} \epsilon^{k}=\frac{1}{2} \Sigma_{\hat{a}}^{\hat{b}} \mathbf{D}_{\hat{b}} \epsilon^{k}+\frac{1}{8}\left(3 w_{\hat{a} \hat{b}} \Gamma^{\hat{b}}+\varepsilon_{\hat{a} \hat{b} \hat{c} \hat{d} \hat{e}} w^{\hat{b} \hat{c}} \Sigma^{\hat{d} \hat{e}}\right) \epsilon^{k}+\frac{1}{4}\left(c_{\hat{a}}{ }^{k} l \mathbb{1}-\frac{1}{2} c^{\hat{b} k}{ }_{l} \Sigma_{\hat{a} \hat{b}}\right) \epsilon^{l} .
$$

This equation may be rewritten in a simpler form if we introduce covariant derivatives with torsion,

$$
\hat{\mathbf{D}}_{\hat{a}}:=\mathbf{D}_{\hat{a}}-\frac{1}{4} c_{\hat{a}}^{p q} J_{p q}-\frac{1}{4} \varepsilon_{\hat{a} \hat{b} \hat{c} \hat{d} \hat{e}} w^{\hat{b} \hat{c}} M^{\hat{d} \hat{e}} .
$$

Then (4.14) turns into

$$
\hat{\mathbf{D}}_{\hat{a}} \epsilon^{k}=-\frac{1}{5} \Gamma_{\hat{a}} \Gamma^{\hat{b}} \hat{\mathbf{D}}_{\hat{b}} \epsilon^{k} .
$$

This is a generalization of the 5D equation for twistor spinors (see, e.g., [40, 41]), which makes use of the torsion-free covariant derivative $\nabla_{\hat{a}}$ (the Levi-Cività connection) instead of $\hat{\mathbf{D}}_{\hat{a}}$.

An important property of twistor spinors is that they 'square' to Killing vector fields $[40,41]$. This property remains valid in our case. Associated with a non-zero commuting spinor $\epsilon^{i}$ is the non-zero real 5-vector

$$
V_{\hat{a}}:=\left(\Gamma_{\hat{a}}\right)^{\hat{\alpha} \hat{\beta}} \varepsilon_{i j} \epsilon_{\hat{\alpha}}^{i} \epsilon_{\hat{\beta}}^{j} .
$$

If $\epsilon^{i}$ is a solution of (4.16), then $V^{\hat{a}}$ is a conformal Killing vector field,

$$
\mathbf{D}_{(\hat{a}} V_{\hat{b})}=\frac{1}{5} \eta_{\hat{a} \hat{b}} \mathbf{D}_{\hat{c}} V^{\hat{c}}
$$

The torsion tensor does not contribute to this relation. It is a short calculation to check that

$$
V^{\hat{a}} V_{\hat{a}}=-F^{2}, \quad F:=\varepsilon^{\hat{\alpha} \hat{\beta}} \varepsilon_{i j} \epsilon_{\hat{\alpha}}^{i} \epsilon_{\hat{\beta}}^{j} .
$$

Since $F$ is real, $V^{\hat{a}}$ is time-like or null. In the spirit of [51], one can construct different bilinears from a commuting conformal Killing spinor. These bilinears and their properties are given in appendix B.

By construction, we have the identities

$$
\delta\left(\mathcal{D}_{\hat{\alpha}}^{i} S^{k l}\right)=0, \quad \delta\left(\mathcal{D}_{\hat{\alpha}}^{i} C_{\hat{a}}^{k l}\right)=0, \quad \delta\left(\mathcal{D}_{\hat{\alpha}}^{i} X_{\hat{a} \hat{b}}\right)=0, \quad \delta\left(\mathcal{D}_{\hat{\alpha}}^{i} N_{\hat{a} \hat{b}}\right)=0,
$$

\footnotetext{
${ }^{6}$ In what follows, we will sometimes avoid writing spinor indices explicitly. In particular, we will denote $\epsilon^{i}:=\epsilon_{\hat{\alpha}}^{i}$, and use $\Gamma_{\hat{a}}$ and $\Sigma_{\hat{a} \hat{b}}$ for $\left(\Gamma_{\hat{a}}\right)_{\hat{\alpha}}^{\hat{\beta}}$ and $\left(\Sigma_{\hat{a} \hat{b}}\right)_{\hat{\alpha}}^{\hat{\beta}}$, respectively.
} 
which imply that the conditions (4.1) are superconformal. Evaluating explicitly the bar-projection of the left-hand sides in (4.20), non-trivial information may be extracted. We derive

$$
\begin{aligned}
& \mathcal{D}_{\hat{\alpha}}^{i} \mathcal{D}^{\hat{\beta}(k} \mathcal{D}_{\hat{\beta}}^{l)} \sigma \mid=\epsilon_{j}^{\hat{\beta}}\left[-2 \mathrm{i}\left[\mathcal{D}_{\hat{\alpha}}^{i}, \mathcal{D}_{\hat{\beta}}^{j}\right] S^{k l} \mid+4 \varepsilon^{i j} \mathbf{D}_{\hat{\alpha} \hat{\beta}} s^{k l}+4 \varepsilon^{i j} c_{\hat{\alpha} \hat{\beta}}{ }^{(k}{ }_{p} s^{l) p}\right. \\
& \left.+8\left(x_{\hat{\alpha} \hat{\beta}}+n_{\hat{\alpha} \hat{\beta}}\right)\left(\varepsilon^{k\left(i s^{j}\right) l}+\varepsilon^{l\left(i s^{j}\right) k}\right)\right] \\
& -12 \mathrm{i} \eta_{\hat{\alpha} j}\left(\varepsilon^{k(i} s^{j) l}+\varepsilon^{l(i} s^{j) k}\right)+4 \mathrm{i} \eta_{\hat{\alpha}}^{i} s^{k l}, \\
& \left(\Gamma_{\hat{a}}\right)^{\hat{\gamma} \hat{\delta}} \mathcal{D}_{\hat{\alpha}}^{i} \mathcal{D}_{\hat{\gamma}}^{(k} \mathcal{D}_{\hat{\delta}}^{l)} \sigma \mid=\epsilon_{j}^{\hat{\beta}}\left[-\mathrm{i}\left[\mathcal{D}_{\hat{\alpha}}^{i}, \mathcal{D}_{\hat{\beta}}^{j}\right] C_{\hat{a}}^{k l} \mid+2 \varepsilon^{i j} \mathbf{D}_{\hat{\alpha} \hat{\beta}} c_{\hat{a}}^{k l}+2 \varepsilon_{\hat{\alpha} \hat{\beta}} \varepsilon^{i j} x_{\hat{a} \hat{d}} c^{\hat{d} k l}\right. \\
& +\frac{1}{2} \varepsilon^{i j} \varepsilon_{\hat{a} \hat{b} \hat{c} \hat{d} \hat{e}}\left(\Gamma^{\hat{b}}\right)_{\hat{\alpha} \hat{\beta}} n^{\hat{c} \hat{d}} c^{\hat{e} k l}-\varepsilon_{\hat{a} \hat{b} \hat{c} \hat{d} \hat{e}}\left(\Sigma^{\hat{b} \hat{c}}\right)_{\hat{\alpha} \hat{\beta}} c^{\hat{d} i j} c^{\hat{e} k l} \\
& -4 s^{i j}\left(\Sigma_{\hat{a} \hat{d}}\right)_{\hat{\alpha} \hat{\beta}} c^{\hat{d} k l}-6 \varepsilon_{\hat{\alpha} \hat{\beta}} \varepsilon^{i j} s^{(k}{ }_{p} c_{\hat{a}}^{l) p}+2 \varepsilon^{i j}\left(\Gamma^{\hat{d}}\right)_{\hat{\alpha} \hat{\beta}} c_{\hat{d}}\left(k{ }_{p} c_{\hat{a}}^{l) p}\right. \\
& \left.+4\left(x_{\hat{\alpha} \hat{\beta}}+n_{\hat{\alpha} \hat{\beta}}\right)\left(\varepsilon^{k(i} c_{\hat{a}}^{j) l}+\varepsilon^{l(i} c_{\hat{a}}^{j) k}\right)\right] \\
& +4 \mathrm{i} \eta^{\hat{\beta} i}\left(\Sigma_{\hat{a} \hat{b}}\right) \hat{\beta} \hat{\alpha} c^{\hat{b} k l}-6 \mathrm{i} \eta_{\hat{\alpha} j}\left(\varepsilon^{k(i} c_{\hat{a}}^{j) l}+\varepsilon^{l(i} c_{\hat{a}}^{j) k}\right)+2 \mathrm{i} \eta_{\hat{\alpha}}^{i} c_{\hat{a}}^{k l}, \\
& \left(\Sigma_{\hat{a} \hat{b}}\right)^{\hat{\beta} \hat{\gamma}} \mathcal{D}_{\hat{\alpha}}^{i} \mathcal{D}_{\hat{\beta}}^{k} \mathcal{D}_{\hat{\gamma} k} \sigma \mid=\epsilon_{j}^{\hat{\beta}}\left[2 \mathrm{i}\left[\mathcal{D}_{\hat{\alpha}}^{i}, \mathcal{D}_{\hat{\beta}}^{j}\right] X_{\hat{a} \hat{b}} \mid-4 \varepsilon^{i j} \mathbf{D}_{\hat{\alpha} \hat{\beta}} x_{\hat{a} \hat{b}}+2 \varepsilon^{i j}\left(\Gamma_{\hat{c}}\right)_{\hat{\alpha} \hat{\beta}} n_{\hat{d} \hat{e}} x_{\hat{f}[\hat{a}} \varepsilon_{\hat{b}]}^{\hat{c} d \hat{e} \hat{f}}\right. \\
& \left.-4\left(\Sigma_{\hat{c} \hat{d}}\right)_{\hat{\alpha} \hat{\beta}} c_{\hat{e}}^{i j} x_{\hat{f}[\hat{a}} \varepsilon_{\hat{b}]}^{\hat{c} \hat{d} \hat{e} \hat{f}}-16 s^{i j}\left(\Sigma_{[\hat{a}}^{\hat{d}}\right)_{\hat{\alpha} \hat{\beta}} x_{\hat{b}] \hat{d}}\right] \\
& +16 \mathrm{i} \eta^{\hat{\beta} i}\left(\Sigma_{[\hat{a}}^{\hat{c}}\right)_{\hat{\beta} \hat{\alpha}} x_{\hat{b}] \hat{c}}-4 \mathrm{i} \eta_{\hat{\alpha}}^{i} x_{\hat{a} \hat{b}}, \\
& \left(\Sigma_{\hat{a} \hat{b}}\right)^{\hat{\beta} \hat{\gamma}} \mathcal{D}_{\hat{\alpha}}^{i} \mathcal{D}_{\hat{\beta}}^{k} \mathcal{D}_{\hat{\gamma} k} \sigma \mid=\epsilon_{j}^{\hat{\beta}}\left[\mathrm{i}\left[\mathcal{D}_{\hat{\alpha}}^{i}, \mathcal{D}_{\hat{\beta}}^{j}\right] N_{\hat{a} \hat{b}} \mid-2 \varepsilon^{i j} \mathbf{D}_{\hat{\alpha} \hat{\beta}} n_{\hat{a} \hat{b}}+4 \varepsilon_{\hat{\alpha} \hat{\beta}} \varepsilon^{i j} x_{[\hat{a}}{ }^{\hat{d}} n_{\hat{b}] \hat{d}}\right. \\
& +\varepsilon^{i j}\left(\Gamma_{\hat{c}}\right)_{\hat{\alpha} \hat{\beta}} n_{\hat{d} \hat{e}} n_{\hat{f}[\hat{a}} \varepsilon_{\hat{b}]}^{\hat{c} \hat{d} \hat{e} \hat{f}}-2\left(\Sigma_{\hat{c} \hat{d}}\right)_{\hat{\alpha} \hat{\beta}} c_{\hat{e}}^{i j} n_{\hat{f}[\hat{a}} \varepsilon_{\hat{b}]} \hat{c} \hat{d} \hat{e} \hat{f} \\
& \left.-8 s^{i j}\left(\Sigma_{[\hat{a}}^{\hat{d}}\right)_{\hat{\alpha} \hat{\beta}} n_{\hat{b}] \hat{d}}\right] \\
& +8 \mathrm{i} \eta^{\hat{\beta} i}\left(\Sigma_{[\hat{a}}^{\hat{c}}\right)_{\hat{\beta} \hat{\alpha}} n_{\hat{b}] \hat{c}}-2 \mathrm{i} \eta_{\hat{\alpha}}^{i} n_{\hat{a} \hat{b}} .
\end{aligned}
$$

These identities become especially useful for those supersymmetric backgrounds which correspond to Poincaré or anti-de Sitter supergravities.

\subsection{Killing spinors}

In the case of Poincaré or anti-de Sitter supergravities, the equations given in the previous subsection have to be supplemented by the additional condition

$$
\sigma[\xi]=0 \quad \Longrightarrow \quad \eta^{i}=0
$$

in accordance with eq. (3.22). Let us remind the reader that we are not yet specifying any particular compensators. However, we are assuming that some compensator has been chosen and the gauge condition (3.20) has been imposed.

Due to eq. (4.22), the equation for conformal Killing spinors, eq. (4.12), turns into

$$
\mathbf{D}_{\hat{a}} \epsilon^{k}=\left(\frac{1}{2} s^{k}{ }_{l} \Gamma_{\hat{a}}+\frac{1}{2} \delta_{l}^{k} x_{\hat{a} \hat{b}} \Gamma^{\hat{b}}-\frac{1}{8} \delta_{l}^{k} \varepsilon_{\hat{a} \hat{b} \hat{c} \hat{d} \hat{e}} n^{\hat{b} \hat{c}} \Sigma^{\hat{d} \hat{e}}-\frac{1}{2} c_{\hat{b}}{ }^{k} \sum_{\hat{a}}{ }^{\hat{b}}\right) \epsilon^{l} .
$$


Its solutions will be called Killing spinors. As demonstrated earlier, associated with a commuting conformal Killing spinor $\epsilon^{k}$ is the conformal Killing vector $V_{\hat{a}}$ defined by eq. (4.17). In the case that $\epsilon^{k}$ is a Killing spinor field, it is simple to prove that $\mathbf{D}_{\hat{a}} V^{\hat{a}}=0$ and hence

$$
\mathbf{D}_{(\hat{a}} V_{\hat{b})}=0 \text {. }
$$

Thus $V_{\hat{a}}$ is a Killing vector field.

\section{Supersymmetric backgrounds: eight supercharges}

The existence of rigid supersymmetries imposes non-trivial restrictions on the background fields in off-shell Poincaré or anti-de Sitter supergravities. For simplicity, here we restrict our analysis to the case of eight supercharges and derive constraints on the geometry.

Since $\sigma[\xi]=0$, the equations (4.21) immediately imply the following conditions:

$$
\left[\mathcal{D}_{\hat{\alpha}}^{i}, \mathcal{D}_{\hat{\beta}}^{j}\right] S^{k l}\left|=0, \quad\left[\mathcal{D}_{\hat{\alpha}}^{i}, \mathcal{D}_{\hat{\beta}}^{j}\right] C_{\hat{a}}^{k l}\right|=0, \quad\left[\mathcal{D}_{\hat{\alpha}}^{i}, \mathcal{D}_{\hat{\beta}}^{j}\right] X_{\hat{a} \hat{b}}\left|=0, \quad\left[\mathcal{D}_{\hat{\alpha}}^{i}, \mathcal{D}_{\hat{\beta}}^{j}\right] N_{\hat{a} \hat{b}}\right|=0 .
$$

The meaning of these conditions is that all dimension-2 auxiliary fields, which belong to the supergravity multiplet, vanish. Information about the background geometry is encoded in the background dimension-1 fields $s^{i j}, c_{\hat{a}}^{i j}, x_{\hat{a} \hat{b}}$ and $n_{\hat{a} \hat{b}}$. The same equations (4.21) also lead to a set of conditions on these tensors. Below we describe the various cases by the values of $s:=\sqrt{\frac{1}{2} s^{i j} s_{i j}}$ and $c_{\hat{a}}^{i j}$.

The relations (5.1) are in fact corollaries of more general results that follow from the following observation. For any background admitting eight supercharges, if there is a tensor superfield $T$ such that its bar-projection vanishes, $T \mid=0$, and this condition is supersymmetric, then the entire superfield is zero, $T=0$. For all supersymmetric backgrounds, the conditions (4.1) hold. Therefore, all backgrounds with eight supercharges should fulfil the superfield conditions

$$
\mathcal{D}_{\hat{\alpha}}^{i} S^{k l}=0, \quad \mathcal{D}_{\hat{\alpha}}^{i} C_{\hat{a}}^{k l}=0, \quad \mathcal{D}_{\hat{\alpha}}^{i} X_{\hat{a} \hat{b}}=0, \quad \mathcal{D}_{\hat{\alpha}}^{i} N_{\hat{a} \hat{b}}=0 .
$$

The relations (5.1) obviously follow from these conditions.

\subsection{The case $s \neq 0$}

When $s \neq 0$, it can be shown that eqs. (4.21) imply the conditions:

$$
\begin{aligned}
\mathbf{D}_{\hat{a}} s^{i j} & =0 & & \Longrightarrow & s & =\text { const }, \\
c_{\hat{a}}{ }^{i j} & =0, & x_{\hat{a} \hat{b}} & =0, & n_{\hat{a} \hat{b}} & =0 .
\end{aligned}
$$

The Killing spinor equation takes the simple form

$$
\mathbf{D}_{\hat{a}} \epsilon^{k}=\frac{1}{2} s^{k}{ }_{l} \Gamma_{\hat{a}} \epsilon^{l} .
$$

By computing $\left[\mathbf{D}_{\hat{a}}, \mathbf{D}_{\hat{b}}\right] \epsilon^{k}$ and using (5.4) together with (5.3) one obtains

$$
\left[\mathbf{D}_{\hat{a}}, \mathbf{D}_{\hat{b}}\right] \epsilon^{k}=-s^{2} \Sigma_{\hat{a} \hat{b}} \epsilon^{k}=\left(\frac{1}{2} \delta_{l}^{k} \mathcal{R}_{\hat{a} \hat{b}}^{\hat{c} \hat{d}} \Sigma_{\hat{c} \hat{d}}+\mathcal{R}_{\hat{a} \hat{b}}^{k} l \mathbb{1}\right) \epsilon^{l},
$$


from which we can read off the expressions for the Lorentz and $\mathrm{SU}(2)$ curvatures $^{7}$

$$
\begin{aligned}
& \mathcal{R}_{\hat{a} \hat{b}}{ }^{\hat{c} \hat{d}}=-2 s^{2} \delta_{[\hat{a}}^{\hat{c}} \delta_{\hat{b}]}^{\hat{d}}, \\
& \mathcal{R}_{\hat{a} \hat{b}}^{k l}=0 \text {. }
\end{aligned}
$$

Hence in this case the supersymmetric background is necessarily 5D anti-de Sitter space, $\mathrm{AdS}_{5}$. It follows from (5.3b) that three dimension-1 superfield torsion tensors vanish,

$$
C_{\hat{a}}^{i j}=0, \quad X_{\hat{a} \hat{b}}=0, \quad N_{\hat{a} \hat{b}}=0 .
$$

The resulting superspace $A d S^{5 / 8}$ and rigid supersymmetric field theories in $\operatorname{Ad} S^{5 / 8}$ have thoroughly been studied in $[18,49]$.

\subsection{The case $s=0$ and $c_{\hat{a}}^{i j} \neq 0$}

If $s=0$ and $c_{\hat{a}}^{i j} \neq 0$, the relations (4.21) imply that some of the background fields vanish,

$$
s^{i j}=0, \quad x_{\hat{a} \hat{b}}=0, \quad n_{\hat{a} \hat{b}}=0,
$$

as well as the following constraints on $c_{\hat{a}}^{i j}$

$$
\mathbf{D}_{\hat{a}} c_{\hat{b}}^{i j}=0, \quad c_{\hat{a}}^{(i}{ }_{k} c_{\hat{b}}^{j) k}=0 .
$$

These constraints tell us that $c_{\hat{a}}^{i j}$ is a composite object being the product of a real 5 -vector $c_{\hat{a}}$ and a real isovector $c^{i j}$ such that $\overline{c^{i j}}=c_{i j}$,

$$
c_{\hat{a}}^{i j}=c_{\hat{a}} c^{i j} .
$$

By rescaling $c_{\hat{a}}$ and $c^{i j}$ we can always make the choice

$$
c^{i j} c_{i j}=2
$$

Then it follows from $\mathbf{D}_{\hat{a}} c_{\hat{b}}^{i j}=0$ that $c_{\hat{a}}$ and $c^{i j}$ are covariantly constant,

$$
\mathbf{D}_{\hat{a}} c^{i j}=0, \quad \mathbf{D}_{\hat{a}} c_{\hat{b}}=0 .
$$

The 5-vector $c^{\hat{a}}$ may be time-like, space-like or null. Since it is covariantly constant, the Lorentz curvature tensor is constrained by

$$
\mathcal{R}_{\hat{a} \hat{b} \hat{b} \hat{d}} c^{\hat{d}}=0 .
$$

For the background under consideration, the Killing spinor equation is

$$
\mathbf{D}_{\hat{a}} \epsilon^{k}=-\frac{1}{2} c^{\hat{b}} c^{k}{ }_{l} \Sigma_{\hat{a} \hat{b}} \epsilon^{l} .
$$

\footnotetext{
${ }^{7}$ Note that here we compute the component curvature tensors by using the Killing spinor equation (5.4). The same results can be read off by bar-projecting the dimension- 2 superspace curvature tensors.
} 
We compute $\left[\mathbf{D}_{\hat{a}}, \mathbf{D}_{\hat{b}}\right] \epsilon^{k}$ by making use of (5.14) in conjunction with eqs. (5.10) and (5.11). The result is

$$
\left[\mathbf{D}_{\hat{a}}, \mathbf{D}_{\hat{b}}\right] \epsilon^{k}=-\frac{1}{2}\left(c_{[\hat{a}} \delta_{\hat{b}]}^{[\hat{c}} c^{\hat{d}]}+\frac{1}{2}\left(c^{\hat{e}} c_{\hat{e}}\right) \delta_{[\hat{a}}^{\hat{c}} \delta_{\hat{b}]}^{\hat{d}}\right) \Sigma_{\hat{c} \hat{d}} \epsilon^{k},
$$

from which we can read off the Lorentz and $\mathrm{SU}(2)$ curvature tensors

$$
\begin{aligned}
& \mathcal{R}_{\hat{a} \hat{b}}^{\hat{c} \hat{d}}=-\left(c_{[\hat{a}} \delta_{\hat{b}]}^{[\hat{c}} c^{\hat{d}]}+\frac{1}{2}\left(c^{\hat{e}} c_{\hat{e}}\right) \delta_{[\hat{a}}^{\hat{c}} \delta_{\hat{b}]}^{\hat{d}}\right), \\
& \mathcal{R}_{\hat{a} \hat{b}} k l=0 .
\end{aligned}
$$

It follows from (5.16a) that the Ricci tensor and the scalar curvature are

$$
\mathcal{R}_{\hat{a} \hat{b}}=\frac{3}{4}\left(c_{\hat{a}} c_{\hat{b}}-\eta_{\hat{a} \hat{b}} c^{\hat{e}} c_{\hat{e}}\right), \quad \mathcal{R}=-3 c^{\hat{e}} c_{\hat{e}} .
$$

As concerns the Weyl tensor

$$
C_{\hat{a} \hat{b} \hat{c} \hat{d}}=\mathcal{R}_{\hat{a} \hat{b} \hat{c} \hat{d}}-\frac{2}{3}\left(\eta_{\hat{a}[\hat{c}} \mathcal{R}_{\hat{d}] \hat{b}}-\eta_{\hat{b}[\hat{c}} \mathcal{R}_{\hat{d}] \hat{a}}\right)+\frac{1}{6} \mathcal{R} \eta_{\hat{a}[\hat{c}} \eta_{\hat{d}] \hat{b}}
$$

it is identically zero for the above background,

$$
C_{\hat{a} \hat{b} \hat{c} \hat{d}}=0
$$

The above supersymmetric backgrounds are generalizations of those found by Festuccia and Seiberg [27] in the case of the old minimal formulation for $4 \mathrm{D} \mathcal{N}=1$ supergravity.

The existence of a covariantly constant vector field $c^{\hat{a}}$ means that spacetime is decomposable in the non-null case (see, e.g., [52]). In this case the space is the product of a fourand a one-dimensional manifold. We can choose a coordinate frame $x^{\hat{m}}=\left(x^{m}, \zeta\right)$, where $m=1,2,3,4$, such that the vector field $c^{\hat{a}} e_{\hat{a}}$ is proportional to $\partial / \partial \zeta$ and the metric reads

$$
\mathrm{d} s_{5}^{2}=g_{m n}\left(x^{r}\right) \mathrm{d} x^{m} \mathrm{~d} x^{n}+\varepsilon(\mathrm{d} \zeta)^{2}=\eta_{a b} e^{a} e^{b}+\varepsilon(\mathrm{d} \zeta)^{2}, \quad e^{a}:=\mathrm{d} x^{m} e_{m}{ }^{a}\left(x^{n}\right),
$$

where $\varepsilon=-1$ when $c^{\hat{a}}$ is time-like, and $\varepsilon=+1$ when $c^{\hat{a}}$ is space-like. The metric $\mathrm{d} s_{4}^{2}=$ $g_{m n}\left(x^{r}\right) \mathrm{d} x^{m} \mathrm{~d} x^{n}$ corresponds to a four-dimensional submanifold $\mathcal{M}^{4}$ orthogonal to $c^{\hat{a}} e_{\hat{a}}$. The identity (5.13) tells us

$$
\mathcal{R}_{\hat{a} \hat{b} \hat{c} \zeta}=0
$$

Let $\mathfrak{R}_{a b c d}$ be the curvature of the submanifold $\mathcal{M}^{4}$. It is clear that

$$
\mathfrak{R}_{a b c d}=\mathcal{R}_{a b c d} .
$$

Then from (5.17) and (5.19) we deduce

$$
\mathfrak{R}_{a b}=-\frac{3}{4} c^{2} \eta_{a b}, \quad \mathfrak{C}_{a b c d}=0,
$$

where $c^{2}=c^{\hat{e}} c_{\hat{e}}$. We conclude that $\mathcal{M}^{4}$ is a four-sphere, $S^{4}$, when $c^{\hat{a}}$ is time-like. In the case that $c^{\hat{a}}$ is space-like, $\mathcal{M}^{4}$ is a four-dimensional anti-de Sitter space, $\operatorname{AdS}_{4}$. 
Finally, if $c^{\hat{a}}$ is null, $c^{\hat{a}} c_{\hat{a}}=0$, it is possible to chose a coordinate system in which the metric reads

$$
\mathrm{d} s^{2}=\mathrm{e}^{u}\left(2 \mathrm{~d} u \mathrm{~d} v+\delta_{i j} \mathrm{~d} x^{i} \mathrm{~d} x^{j}\right), \quad i, j=1,2,3,
$$

with $c^{\hat{a}} e_{\hat{a}} \propto \partial / \partial v$. This is a special example of pp-waves, see, e.g., [53].

In conclusion, we present those superspace geometries that generate the supersymmetric backgrounds given. It follows from (5.8) that $S^{i j}=0, X_{\hat{a} \hat{b}}=0$ and $N_{\hat{a} \hat{b}}=0$. The superspace geometry is described by a single covariantly constant tensor $C_{\hat{a}}^{i j}, \mathcal{D}_{\hat{B}} C_{\hat{a}}^{i j}=0$. The algebra of covariant derivatives is

$$
\begin{aligned}
\left\{\mathcal{D}_{\hat{\alpha}}^{i}, \mathcal{D}_{\hat{\beta}}^{j}\right\} & =-2 \mathrm{i} \varepsilon^{i j} \mathcal{D}_{\hat{\alpha} \hat{\beta}}-\frac{\mathrm{i}}{2} \varepsilon^{\hat{a} \hat{b} \hat{c} \hat{d} \hat{e}}\left(\Sigma_{\hat{a} \hat{b}}\right)_{\hat{\alpha} \hat{\beta}} C_{\hat{c}}^{i j} M_{\hat{d} \hat{e}}-\mathrm{i} \varepsilon^{i j} C_{\hat{\alpha} \hat{\beta}}^{k l} J_{k l}, \\
{\left[\mathcal{D}_{\hat{a}}, \mathcal{D}_{\hat{\beta}}^{j}\right] } & =\frac{1}{2}\left(\Sigma_{\hat{a}}^{\hat{b}}\right)_{\hat{\beta}}^{\hat{\gamma}} C_{\hat{b}}^{j}{ }_{k} \mathcal{D}_{\hat{\gamma}}^{k}, \\
{\left[\mathcal{D}_{\hat{a}}, \mathcal{D}_{\hat{b}}\right] } & =\frac{1}{4}\left(\delta_{[\hat{a}}^{[\hat{c}} C_{\hat{b}] k l} C^{\hat{d}] k l}-\frac{1}{2} \delta_{[\hat{a}}^{\hat{c}} \delta_{\hat{b}]}^{\hat{d}} C^{\hat{e} k l} C_{\hat{e} k l}\right) M_{\hat{c} \hat{d}} .
\end{aligned}
$$

Integrability condition for the constraint $\mathcal{D}_{\hat{\alpha}}^{i} C_{\hat{b}}^{j k}=0$ is

$$
C_{\hat{a}}^{(i}{ }_{k} C_{\hat{b}}^{j) k}=0 \text {. }
$$

It implies that the superfield $C_{\hat{a}}^{i j}$ factorizes,

$$
C_{\hat{a}}^{i j}=C_{\hat{a}} C^{i j}, \quad C^{i j} C_{i j}=2 .
$$

The condition that $C_{\hat{a}}{ }^{i j}$ is covariantly constant is equivalent to $C_{i j} \mathcal{D}_{\hat{A}} C_{\hat{b}}=-C_{\hat{b}} \mathcal{D}_{\hat{A}} C_{i j}$, which leads to

$$
2 \mathcal{D}_{\hat{A}} C_{\hat{b}}=-C_{\hat{b}} C^{i j} \mathcal{D}_{\hat{A}} C_{i j}=-\frac{1}{2} C_{\hat{b}} \mathcal{D}_{\hat{A}}\left(C^{i j} C_{i j}\right)=0 .
$$

Thus both tensors $C_{\hat{a}}$ and $C^{i j}$ are covariantly constant,

$$
\mathcal{D}_{\hat{A}} C_{\hat{b}}=0, \quad \mathcal{D}_{\hat{A}} C^{i j}=0 .
$$

Because the superspace is conformally flat, the isometry superalgebra is a subalgebra of the $5 \mathrm{D}$ superconformal algebra $\mathfrak{f}(4)$.

\subsection{The case $s=0$ and $c_{\hat{a}}^{i j}=0$}

It remains to consider the case

$$
s^{i j}=0, \quad c_{\hat{a}}^{i j}=0 .
$$

Here the relations (4.21) imply the following constraints on $x_{\hat{a} \hat{b}}$ and $n_{\hat{a} \hat{b}}$

$$
\begin{aligned}
\mathbf{D}_{\hat{a}} x_{\hat{b} \hat{c}} & =\frac{1}{2} \varepsilon_{\hat{a} \hat{d} \hat{e} \hat{f}[\hat{b}} x_{\hat{c}]} n^{\hat{d}} n^{\hat{e} \hat{f}}, \\
\mathbf{D}_{\hat{a}} n_{\hat{b} \hat{c}} & =\frac{1}{2} \varepsilon_{\hat{a} \hat{d} \hat{e} \hat{f}[\hat{b}} n_{\hat{c}]}{ }^{\hat{d}} n^{\hat{e} \hat{f}}=-\frac{1}{8} \eta_{\hat{a}[\hat{b}} \varepsilon_{\hat{b}] \hat{d} \hat{e} \hat{f} \hat{g}} n^{\hat{d} \hat{e}} n^{\hat{f} \hat{g}}, \\
x_{[\hat{a}}{ }^{\hat{c}} n_{\hat{b}] \hat{c}} & =0 .
\end{aligned}
$$


The constraint (5.31c) can be rewritten in a matrix form as follows:

$$
[\hat{x}, \hat{n}]=0, \quad \hat{x}:=\left(x_{\hat{a}}^{\hat{b}}\right), \quad \hat{n}:=\left(n_{\hat{a}}^{\hat{b}}\right) .
$$

An important consequence of the constraints (5.31a) and (5.31c) is that $x_{\hat{a} \hat{b}}$ is a closed two-form,

$$
\mathbf{D}_{[\hat{a}} x_{\hat{b} \hat{c}]}=0 .
$$

It is a consequence of (5.31b) that $n_{\hat{a} \hat{b}}$ is also a closed two-form,

$$
\mathbf{D}_{[\hat{a}} n_{\hat{b} \hat{c}]}=0 \text {. }
$$

Introducing the Hodge dual of $n_{\hat{a} \hat{b}}$ in the standard way $* n_{\hat{a} \hat{b} \hat{c}}:=\frac{1}{2} \varepsilon_{\hat{a} \hat{b} \hat{c} \hat{d} \hat{e}} n^{\hat{d} \hat{e}}$, the constraint (5.31b) becomes

$$
\mathbf{D}_{\hat{a}} * n_{\hat{b} \hat{c} \hat{d}}=-\frac{3}{2} n_{[\hat{a} \hat{b}} n_{\hat{c} \hat{d}]} .
$$

This relation implies the equation of motion that is derived from a U(1) Chern-Simons action.

For the background under consideration, the Killing spinor equation (4.23) takes the form

$$
\mathbf{D}_{\hat{a}} \epsilon^{k}=\left(\frac{1}{2} \delta_{l}^{k} x_{\hat{a} \hat{b}} \hat{\Gamma}^{\hat{b}}-\frac{1}{8} \delta_{l}^{k} \varepsilon_{\hat{a} \hat{b} \hat{c} \hat{d} \hat{e}} n^{\hat{b} \hat{c}} \Sigma^{\hat{d} \hat{e}}\right) \epsilon^{l} .
$$

We can now compute $\left[\mathbf{D}_{\hat{a}}, \mathbf{D}_{\hat{b}}\right] \epsilon^{k}$ by using (5.36) together with the relations (5.31a)-(5.31c). The result is

$$
\begin{aligned}
{\left[\mathbf{D}_{\hat{a}}, \mathbf{D}_{\hat{b}}\right] \epsilon^{k}=(} & -\frac{3}{4} n_{[\hat{a}}^{[\hat{c}} n_{\hat{b}]}^{\hat{d}]}+\frac{1}{4} n_{\hat{a} \hat{b}} n^{\hat{c} \hat{d}}+\frac{1}{2} \delta_{[\hat{a}}^{[\hat{c}} n_{\hat{b}]} n^{\hat{d}]} n^{\hat{e}}-\frac{1}{8} n_{\hat{e} \hat{f}} n^{\hat{e} \hat{f}} \delta_{[\hat{a}}^{[\hat{c}} \delta_{\hat{b}]}^{\hat{d}]} \\
& \left.+x_{[\hat{a}}^{[\hat{c}} x_{\hat{b}]}^{\hat{d}]}\right) \Sigma_{\hat{c} \epsilon^{k}} \epsilon^{k}
\end{aligned}
$$

From here we read off the Lorentz and SU(2) curvature tensors

$$
\begin{aligned}
& \mathcal{R}_{\hat{a} \hat{b}}^{\hat{c} \hat{d}}=2 x_{[\hat{a}}^{[\hat{c}} x_{\hat{b}]}^{\hat{d}]}+\frac{1}{2} n_{\hat{a} \hat{b}} n^{\hat{c} \hat{d}}-\frac{3}{2} n_{[\hat{a}}^{[\hat{c}} n_{\hat{b}]}^{\hat{d}]}+\delta_{[\hat{a}}^{[\hat{c}} n_{\hat{b}] \hat{e}} n^{\hat{d}] \hat{e}}-\frac{1}{4} n_{\hat{e} \hat{f}} n^{\hat{e} \hat{f}} \delta_{[\hat{a}}^{[\hat{c}} \delta_{\hat{b}]}^{\hat{d}]}, \\
& \mathcal{R}_{\hat{a} \hat{b}} k l=0
\end{aligned}
$$

Actually there is another important constraint on the dimension- 1 tensors $x_{\hat{a} \hat{b}}$ and $n_{\hat{a} \hat{b}}$. For the background under consideration, it can be proved that the dimension-2 superspace Bianchi identities imply the following quadratic equation

$$
x_{[\hat{a} \hat{b}} x_{\hat{c}] \hat{d}}=n_{[\hat{a} \hat{b}} n_{\hat{c}] \hat{d}} \quad \Longleftrightarrow \quad x_{[\hat{a} \hat{b}} x_{\hat{c} \hat{d}]}=n_{[\hat{a} \hat{b}} n_{\hat{c} \hat{d}]} .
$$

This constraint may be seen to be equivalent to the requirement that the Lorentz curvature (5.38a) satisfies the Bianchi identity $\mathcal{R}_{[\hat{a} \hat{b} \hat{c}] \hat{d}}=0$. With the aid of (5.39) we can rewrite the Lorentz curvature in the equivalent form:

$$
\begin{aligned}
& \mathcal{R}_{\hat{a} \hat{b}}^{\hat{c} \hat{d}}=-\frac{1}{6} n_{\hat{a} \hat{b}} n^{\hat{c} \hat{d}}-\frac{1}{6} n_{[\hat{a}}^{[\hat{c}} n_{\hat{b}]}^{\hat{d}]}+\delta_{[\hat{a}}^{[\hat{c}} n_{\hat{b}]} \hat{e}^{\hat{n}} n^{\hat{d}] \hat{e}}-\frac{1}{4} n_{\hat{e} \hat{f}} n^{\hat{e} \hat{f}} \delta_{[\hat{a}}^{[c} \delta_{\hat{b}]}^{\hat{d}]} \\
& +\frac{2}{3} x_{\hat{a} \hat{b}} x^{\hat{c} \hat{d}}+\frac{2}{3} x_{[\hat{a}}^{[\hat{c}} x_{\hat{b}]}^{\hat{d}]} \text {. }
\end{aligned}
$$


The Ricci tensor and the scalar curvature are, respectively,

$$
\begin{aligned}
\mathcal{R}_{\hat{a} \hat{b}} & =x_{\hat{a}}{ }^{\hat{c}} x_{\hat{b} \hat{c}}+\frac{1}{2} n_{\hat{a}}{ }^{\hat{c}} n_{\hat{b} \hat{c}}-\frac{1}{4} n_{\hat{a} \hat{b}} n^{\hat{c} \hat{d}} n_{\hat{c} \hat{d}}, \\
\mathcal{R} & =x^{\hat{a} \hat{b}} x_{\hat{a} \hat{b}}-\frac{3}{4} n^{\hat{a} \hat{b}} n_{\hat{a} \hat{b}} .
\end{aligned}
$$

The Weyl tensor is

$$
\begin{aligned}
& C_{\hat{a} \hat{b} \hat{c} \hat{d}}=-\frac{1}{6}\left(n_{\hat{a} \hat{b}} n_{\hat{c} \hat{d}}-n_{\hat{c}[\hat{a}} n_{\hat{b}] \hat{d}}-\eta_{\hat{c}[\hat{a}} n_{\hat{b}]} n_{\hat{d} \hat{e}}+\eta_{\hat{d}[\hat{a}} n_{\hat{b}]}^{\hat{e}} n_{\hat{c} \hat{e}}+\frac{1}{4} \eta_{\hat{a}[\hat{c}} \eta_{\hat{d}] \hat{b}} n^{\hat{e} \hat{f}} n_{\hat{e} \hat{f}}\right) \\
& +\frac{2}{3}\left(x_{\hat{a} \hat{b}} x_{\hat{c} \hat{d}}-x_{\hat{c}[\hat{a}} x_{\hat{b}] \hat{d}}-\eta_{\hat{c}[\hat{a}} x_{\hat{b}]}{ }^{\hat{e}} x_{\hat{d} \hat{e}}+\eta_{\hat{d}[\hat{a}} x_{\hat{b}]} x_{\hat{c} \hat{e}}+\frac{1}{4} \eta_{\hat{a}[\hat{c}} \eta_{\hat{d}] \hat{b}} x^{\hat{e} \hat{f}} x_{\hat{e} \hat{f}}\right) .
\end{aligned}
$$

An important observation is in order. It may be seen that the Weyl tensor (5.42) vanishes (and the spacetime is conformally flat), $C_{\hat{a} \hat{b} \hat{c} \hat{d}}=0$, under the condition

$$
w_{\hat{a} \hat{b}}:=W_{\hat{a} \hat{b}} \mid=0 \quad \Longleftrightarrow \quad n_{\hat{a} \hat{b}}=2 x_{\hat{a} \hat{b}} .
$$

Due to (5.39), in this case we should also have the condition $x_{[\hat{a} \hat{b}} x_{\hat{c} \hat{d}]}=0$, which is equivalent to the fact that $x_{\hat{a} \hat{b}}$ is a decomposable bivector, $x_{\hat{a} \hat{b}}=u_{[\hat{a}} v_{\hat{b}]}$, for some 5 -vectors $u_{\hat{a}}$ and $v_{\hat{a}}$. Then we deduce from (5.31) that the two-form $x_{\hat{b} \hat{c}}$ is covariantly constant,

$$
\mathbf{D}_{\hat{a}} x_{\hat{b} \hat{c}}=0 \text {. }
$$

We now present the superspace geometry that generates the bosonic background described. In accordance with (5.30), the dimension-1 torsion tensors $S^{i j}$ and $C_{\hat{a}}{ }^{i j}$ vanish. The superspace geometry is determined by the tensors $X_{\hat{a} \hat{b}}$ and $N_{\hat{a} \hat{b}}$ obeying the differential constraints

$$
\begin{aligned}
& \mathcal{D}_{\hat{\alpha}}^{i} X_{\hat{a} \hat{b}}=0, \quad \mathcal{D}_{\hat{\alpha}}^{i} N_{\hat{a} \hat{b}}=0, \\
& \mathcal{D}_{\hat{a}} X_{\hat{b} \hat{c}}=\frac{1}{2} \varepsilon_{\hat{a} \hat{d} \hat{e} \hat{f}[\hat{b}} X_{\hat{c}]}{ }^{\hat{d}} N^{\hat{e} \hat{f}}, \\
& \mathcal{D}_{\hat{a}} N_{\hat{b} \hat{c}}=\frac{1}{2} \varepsilon_{\hat{a} \hat{d} \hat{e} \hat{f}[\hat{b}} N_{\hat{c}]} N^{\hat{d}} N^{\hat{e} \hat{f}}=-\frac{1}{8} \eta_{\hat{a}[\hat{b}} \varepsilon_{\hat{c}] \hat{e} \hat{d} \hat{f} \hat{g}} N^{\hat{d} \hat{e}} N^{\hat{f} \hat{g}}
\end{aligned}
$$

and the algebraic ones

$$
X_{[\hat{a}}^{\hat{c}} N_{\hat{b}] \hat{c}}=0, \quad X_{[\hat{a} \hat{b}} X_{\hat{c}] \hat{d}}=N_{[\hat{a} \hat{b}} N_{\hat{c}] \hat{d}} .
$$

The algebra of covariant derivatives is

$$
\begin{aligned}
\left\{\mathcal{D}_{\hat{\alpha}}^{i}, \mathcal{D}_{\hat{\beta}}^{j}\right\}= & -2 \mathrm{i} \varepsilon^{i j} \mathcal{D}_{\hat{\alpha} \hat{\beta}}-\mathrm{i} \varepsilon_{\hat{\alpha} \hat{\beta}} \varepsilon^{i j} X^{\hat{c} \hat{d}} M_{\hat{c} \hat{d}}+\frac{\mathrm{i}}{4} \varepsilon^{i j} \varepsilon^{\hat{a} \hat{b} \hat{c} \hat{d} \hat{e}}\left(\Gamma_{\hat{a}}\right)_{\hat{\alpha} \hat{\beta}} N_{\hat{b} \hat{c}} M_{\hat{d} \hat{e}} \\
& -4 \mathrm{i}\left(X_{\hat{\alpha} \hat{\beta}}+N_{\hat{\alpha} \hat{\beta}}\right) J^{i j} \\
{\left[\mathcal{D}_{\hat{a}}, \mathcal{D}_{\hat{\beta}}^{j}\right]=} & -\frac{1}{2}\left(X_{\hat{a} \hat{b}}\left(\Gamma^{\hat{b}}\right)_{\hat{\beta}}^{\hat{\gamma}}+\frac{1}{4} \varepsilon_{\hat{a} \hat{b} \hat{c} \hat{d} \hat{e}} N^{\left.\hat{d} \hat{e}\left(\Sigma^{\hat{b} \hat{c}}\right)_{\hat{\beta}}^{\hat{\gamma}}\right) \mathcal{D}_{\hat{\gamma}}^{j}}\right. \\
{\left[\mathcal{D}_{\hat{a}}, \mathcal{D}_{\hat{b}}\right]=} & -\frac{1}{2}\left(\frac{1}{6} N_{\hat{a} \hat{b}} N^{\hat{c} \hat{d}}+\frac{1}{6} N_{[\hat{a}}^{[\hat{c}} N_{\hat{b}]}^{\hat{d}]}-\delta_{[\hat{a}}^{[\hat{c}} N_{\hat{b}] \hat{e}} N^{\hat{d}] \hat{e}}+\frac{1}{4} N_{\hat{e} \hat{f}} N^{\hat{e} \hat{f}} \delta_{[\hat{a}}^{[\hat{c}} \delta_{\hat{b}]}^{\hat{d}]}\right. \\
& \left.-\frac{2}{3} X_{\hat{a} \hat{b}} X^{\hat{c} \hat{d}}-\frac{2}{3} X_{[\hat{a}}^{[\hat{c}} X_{\hat{b}]}^{\hat{d}]}\right) M_{\hat{c} \hat{d}} \cdot
\end{aligned}
$$


This superspace is conformally flat only if $W_{\hat{a} \hat{b}}=X_{\hat{a} \hat{b}}-\frac{1}{2} N_{\hat{a} \hat{b}}=0$, and then the bivector $X_{\hat{a} \hat{b}}$ is covariantly constant and decomposable,

$$
W_{\hat{a} \hat{b}}=0 \quad \Longrightarrow \quad \mathcal{D}_{\hat{A}} X_{\hat{a} \hat{b}}=0, \quad X_{[\hat{a} \hat{b}} X_{\hat{c}] \hat{d}}=0
$$

\section{Vector multiplet compensator}

Up to now we have not specified any conformal compensator. Similar to the case of $4 \mathrm{D} \mathcal{N}=2$ supergravity reviewed in [50], two conformal compensators are required in 5D minimal supergravity. One of them is universally a vector multiplet, while there are several choices for the second compensator. It may be an $\mathcal{O}(2)$ multiplet, or a hypermultiplet, or a nonlinear multiplet. The dilaton Weyl multiplet automatically includes one compensator, an on-shell vector multiplet. In the remainder of this paper, we will study the restrictions on supersymmetric backgrounds which arise when one or two compensators are turned on.

As mentioned above, one of the compensators is invariably an Abelian vector multiplet. The standard way to formulate it is to use gauge covariant derivatives

$$
\mathcal{D}_{\hat{A}}=\mathcal{D}_{\hat{A}}+\mathrm{i} \mathcal{V}_{\hat{A}} \mathfrak{Z}
$$

where $\mathfrak{Z}$ denotes the $\mathrm{U}(1)$ generator and $\mathcal{V}_{\hat{A}}$ is the corresponding connection. In general the gauge covariant derivatives have (anti-)commutation relations

$$
\left[\mathcal{D}_{\hat{A}}, \mathcal{D}_{\hat{B}}\right\}=T_{\hat{A} \hat{B}}^{\hat{C}} \mathcal{D}_{\hat{C}}+\frac{1}{2} R_{\hat{A} \hat{B}}^{\hat{c} \hat{d}} M_{\hat{c} \hat{d}}+R_{\hat{A} \hat{B}}^{k l} J_{k l}+\mathrm{i} F_{\hat{A} \hat{B}} \mathfrak{Z}
$$

in which the torsion, and the Lorentz and $\mathrm{SU}(2)$ curvature tensors are the same as before. In order to describe the vector multiplet, the $\mathrm{U}(1)$ field strength $F_{\hat{A} \hat{B}}$ is constrained such that its components are [3]

$$
\begin{aligned}
& F_{\hat{\alpha} \hat{\beta}}^{i j}=-2 \mathrm{i} \varepsilon^{i j} \varepsilon_{\hat{\alpha} \hat{\beta}} W, \quad F_{\hat{a} \hat{\beta}}^{j}=\left(\Gamma_{\hat{a}}\right)_{\hat{\beta}}{ }_{\hat{\gamma}} \mathcal{D}_{\hat{\gamma}}^{j} W, \\
& F_{\hat{a} \hat{b}}=X_{\hat{a} \hat{b}} W+\frac{\mathrm{i}}{4}\left(\Sigma_{\hat{a} \hat{b}}\right)^{\hat{\gamma} \hat{\delta}} \mathcal{D}_{\hat{\gamma}}^{k} \mathcal{D}_{\hat{\delta} k} W .
\end{aligned}
$$

Here the field strength $W$ is real, $\bar{W}=W$, and obeys the Bianchi identity

$$
\mathcal{D}_{\hat{\alpha}}^{(i} \mathcal{D}_{\hat{\beta}}^{j)} W-\frac{1}{4} \varepsilon_{\hat{\alpha} \hat{\beta}} \mathcal{D}^{\hat{\gamma}(i} \mathcal{D}_{\hat{\gamma}}^{j)} W=\frac{\mathrm{i}}{2} C_{\hat{\alpha} \hat{\beta}}{ }^{i j} W .
$$

The super-Weyl transformation law of the field strength $W$ is

$$
\delta_{\sigma} W=\sigma W
$$

We require the field strength $W$ to be nowhere vanishing, $W>0$, so that it can be used as a conformal compensator. Actually, since $W$ is a Lorentz and $\mathrm{SU}(2)$ scalar superfield, it can be identified with the compensating superfield $\Phi$ introduced in section 3.3. Choosing the super-Weyl gauge

$$
W=1,
$$


completely fixes the super-Weyl gauge freedom. This gauge choice leads to the following restrictions on the dimension- 1 torsion superfields:

$$
X_{\hat{a} \hat{b}}=F_{\hat{a} \hat{b}}, \quad C_{\hat{a}}^{k l}=0 .
$$

The superspace geometry described by the gauge covariant derivatives $\mathcal{D}_{\hat{A}}$ and subject to the condition (6.6) corresponds to the $5 \mathrm{D} \mathcal{N}=1$ minimal supergravity multiplet. It was discovered by Howe [13] in 1982 in the superspace setting and then was fully elaborated in $[1,2]$. In the component approach, the minimal multiplet was rediscovered by Zucker in 1999 [4-6].

\subsection{Supersymmetric backgrounds}

All information about the supersymmetric backgrounds that correspond to the minimal supergravity multiplet can be extracted from the results in sections 4 and 5 . It suffices to take into account the conditions (6.6) and (6.7). In particular, the Killing spinor equation (4.23) turns into

$$
\mathbf{D}_{\hat{a}} \epsilon^{k}=\left(\frac{1}{2} \Gamma_{\hat{a}} s^{k}{ }_{l}+\frac{1}{2} \delta_{l}^{k} f_{\hat{a} \hat{b}} \Gamma^{\hat{b}}-\frac{1}{8} \delta_{l}^{k} \varepsilon_{\hat{a} \hat{b} \hat{c} \hat{d} \hat{e}} n^{\hat{b} \hat{c}} \Sigma^{\hat{d} \hat{e}}\right) \epsilon^{l},
$$

where we have denoted $f_{\hat{a} \hat{b}}:=F_{\hat{a} \hat{b}} \mid=x_{\hat{a} \hat{b}}$. By construction, the two-form $f_{\hat{a} \hat{b}}$ is a U(1) field strength, $\mathbf{D}_{[\hat{a}} f_{\hat{b} \hat{c}]}=0$.

All supersymmetric backgrounds with eight supercharges are characterized by the conditions

$$
f_{\hat{a} \hat{b}} s^{i j}=0, \quad n_{\hat{a} \hat{b}} s^{i j}=0 .
$$

The background fields obey the following differential and algebraic conditions:

$$
\begin{aligned}
& \mathbf{D}_{\hat{a}} s^{k l}=0, \\
& \mathbf{D}_{\hat{a}} f_{\hat{b} \hat{c}}=\frac{1}{2} \varepsilon_{\hat{a} \hat{d} \hat{e} \hat{f}[\hat{b}} f_{\hat{c}]} \hat{d}^{\hat{e} \hat{f}},
\end{aligned}
$$

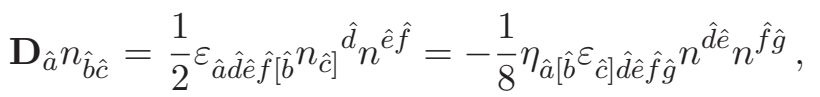

$$
\begin{aligned}
& f_{[\hat{a}}{ }^{\hat{c}} n_{\hat{b}] \hat{c}}=0 \text {, } \\
& f_{[\hat{a} \hat{b}} f_{\hat{c}] \hat{d}}=n_{[\hat{a} \hat{b}} n_{\hat{c}] \hat{d}} \quad \Longleftrightarrow \quad f_{[\hat{a} \hat{b}} f_{\hat{c} \hat{d}]}=n_{[\hat{a} \hat{b}} n_{\hat{c} \hat{d}]} .
\end{aligned}
$$

The curvature tensors can be read off from the results of the previous section by setting $c_{\hat{a}}^{i j}=0$ and $x_{\hat{a} \hat{b}}=f_{\hat{a} \hat{b}}$.

\subsection{The dilaton Weyl multiplet}

In the superspace setting of [3], the so-called dilaton Weyl multiplet [7-12] is realized as the Weyl multiplet coupled to an Abelian vector multiplet such that its field strength $W$ is nowhere vanishing, $W \neq 0$, and enjoys the equation

$$
\mathbb{H}^{i j}=0,
$$


where $\mathbb{H}^{i j}$ denotes the following real isovector [3]

$$
\mathbb{H}^{i j}=\mathrm{i} \mathcal{D}^{\hat{\alpha}(i} W \mathcal{D}_{\hat{\alpha}}^{j)} W+\frac{\mathrm{i}}{2} W \mathcal{D}^{i j} W-2 S^{i j} W^{2}=\frac{\mathrm{i}}{6 W}\left(\mathcal{D}^{i j}+12 \mathrm{i} S^{i j}\right) W^{3},
$$

which is constrained by

$$
\mathcal{D}_{\hat{\alpha}}^{(i} \mathbb{H}^{j k)}=0 .
$$

This constraint defines an $\mathcal{O}(2)$ multiplet. ${ }^{8}$ The super-Weyl transformation law of $\mathbb{H}^{i j}$ is

$$
\delta_{\sigma} \mathbb{H}^{i j}=3 \sigma \mathbb{H}^{i j}
$$

Eq. (6.11) is equivalent to

$$
S^{i j}=\frac{\mathrm{i}}{2 W^{2}}\left\{\mathcal{D}^{\hat{\alpha}(i} W \mathcal{D}_{\hat{\alpha}}^{j)} W+\frac{1}{2} W \mathcal{D}^{i j} W\right\} .
$$

Similar to the rigid supersymmetric case [48], eq. (6.11) originates as the equation of motion in a Chern-Simons model for the vector multiplet.

In the super-Weyl gauge (6.6), we have the condition

$$
S^{i j}=0
$$

in addition to the superfield requirements (6.7).

\section{$\begin{array}{lll}7 & \mathcal{O}(2) & \text { multiplet compensator }\end{array}$}

There are several ways to choose the second supergravity compensator. Similar to the situation in $4 \mathrm{D} \mathcal{N}=2$ supergravity (see, e.g., [50] for a review), one of the most popular choices is a real $\mathcal{O}(2)$ multiplet. ${ }^{9}$ Within the superspace approach of [18], this multiplet is described by an isovector superfield $H^{i j}=H^{j i}=\varepsilon^{i k} \varepsilon^{j l} \overline{H^{k l}}$ which is constrained by

$$
\mathcal{D}_{\hat{\alpha}}^{(i} H^{j k)}=0
$$

and has the super-Weyl transformation law

$$
\delta_{\sigma} H^{i j}=3 \sigma H^{i j}
$$

It is assumed that $H^{i j}$ is nowhere vanishing, $H^{2}:=\frac{1}{2} H^{i j} H_{i j}>0$. The super-Weyl gauge freedom may be used to impose the gauge condition

$$
H^{2}=1 \Longleftrightarrow H_{k}^{i} H_{j}^{k}=-\delta_{j}^{i},
$$

which completely fixes the super-Weyl invariance. Now the analyticity constraint (7.1) and gauge condition (7.3) tell us that $H^{i j}$ is annihilated by all the spinor covariant derivatives,

$$
\mathcal{D}_{\hat{\alpha}}^{i} H^{j k}=0 .
$$

\footnotetext{
${ }^{8}$ In the rigid supersymmetric case, the composite $\mathcal{O}(2)$ multiplet (6.12) was introduced in [48].

${ }^{9}$ It is a $5 \mathrm{D}$ analogue of the $4 \mathrm{D} \mathcal{N}=2$ improved tensor multiplet [54, 55].
} 
This is consistent under the integrability condition $\left\{\mathcal{D}_{\hat{\alpha}}^{i}, \mathcal{D}_{\hat{\beta}}^{j}\right\} H^{k l}=0$, which leads to the following set of constraints:

$$
\begin{aligned}
S^{i j} & =S H^{i j}, \\
N_{\hat{a} \hat{b}} & =-X_{\hat{a} \hat{b}}, \\
\mathcal{D}_{\hat{a}} H^{i j} & =C_{\hat{a}}^{k(i} H^{j)}{ }_{k},
\end{aligned}
$$

for some scalar superfield $S$.

\subsection{Supersymmetric backgrounds}

It is of interest to study those supersymmetric backgrounds which support the curved superspace geometry just described. All information about such backgrounds can be extracted from the results derived in sections 4 and 5 provided we take into account the additional conditions (7.3)-(7.5). The Killing spinor equation (4.23) turns into

$$
\mathbf{D}_{\hat{a}} \epsilon^{k}=\left(\frac{1}{2} s h^{k}{ }_{l} \Gamma_{\hat{a}}+\frac{1}{2} \delta_{l}^{k} x_{\hat{a} \hat{b}} \Gamma^{\hat{b}}+\frac{1}{8} \delta_{l}^{k} \varepsilon_{\hat{a} \hat{b} \hat{c} \hat{d} \hat{e}} x^{\hat{b} \hat{c}} \Sigma^{\hat{d} \hat{e}}-\frac{1}{2} c^{\hat{b} k}{ }_{l} \Sigma_{\hat{a} \hat{b}}\right) \epsilon^{l}
$$

where we have introduced the component fields

$$
s:=S\left|, \quad h^{i j}:=H^{i j}\right|
$$

and used the component relations

$$
s^{i j}=s h^{i j}, \quad n_{\hat{a} \hat{b}}=-x_{\hat{a} \hat{b}},
$$

which follow from (7.5a) and (7.5b). The isovector field is constrained by

$$
h_{k}^{i} h_{j}^{k}=-\delta_{j}^{i}, \quad \mathbf{D}_{\hat{a}} h^{i j}=c_{\hat{a}}^{k(i} h^{j)}{ }_{k}
$$

\subsection{Supersymmetric backgrounds with eight supercharges}

Different maximally supersymmetric backgrounds appear depending on whether the fields $s$ and/or $c_{\hat{a}}^{i j}$ are zero or not. In fact, there are three cases: (i) $s \neq 0$; (ii) $c_{\hat{a}}{ }^{i j} \neq s=0$; and (iii) $s=c_{\hat{a}}^{i j}=0$. They correspond to those worked out in sections 5.1, 5.2 and 5.3, respectively. The choice of the real $\mathcal{O}(2)$ multiplet compensator requires that we take into account the additional relations (7.8) and (7.9). It is then straightforward to read off the curvatures and Weyl tensors from the corresponding ones in section 5.

\section{Off-shell supergravity}

We turn to an off-shell formulation for 5D minimal supergravity obtained by coupling the Weyl multiplet to the following compensators: (i) the vector multiplet; and (ii) the $\mathcal{O}(2)$ multiplet. This is the $5 \mathrm{D}$ analogue of the off-shell formulation for $4 \mathrm{D} \mathcal{N}=2$ supergravity proposed by de Wit, Philippe and Van Proeyen [54]. Our goal is to elucidate those restrictions on the supersymmetric backgrounds that follow from the structure of the compensators chosen. 
As has been discussed above, the super-Weyl gauge freedom may be fixed using one of the two compensators, either by imposing the condition $W=1$ or the alternative one $H=1$. To start with, we do not impose any super-Weyl condition and list those off-shell relations which turn into non-trivial constraints upon imposing a super-Weyl gauge.

In the case of the vector compensator, the Bianchi identity (6.4) can be interpreted as an equation that expresses $C_{\hat{a}}^{k l}$ in terms of $W$ :

$$
C_{\hat{a}}^{i j}=\frac{\mathrm{i}}{2 W}\left(\Gamma_{\hat{a}}\right)^{\hat{\alpha} \hat{\beta}} \mathcal{D}_{\hat{\alpha}}^{(i} \mathcal{D}_{\hat{\beta}}^{j)} W .
$$

It is also useful to rewrite equation $(6.3 \mathrm{~b})$ as

$$
X_{\hat{a} \hat{b}}=\frac{1}{W}\left(F_{\hat{a} \hat{b}}-\frac{\mathrm{i}}{4}\left(\Sigma_{\hat{a} \hat{b}}\right)^{\hat{\gamma} \hat{\delta}} \mathcal{D}_{\hat{\gamma}}^{k} \mathcal{D}_{\hat{\delta} k} W\right) .
$$

The relation expresses the torsion superfields $X_{\hat{a} \hat{b}}$ in terms of the vector multiplet. In the super-Weyl gauge $W=1$, the relations (8.1) take the form (6.7).

In the case of the $\mathcal{O}(2)$ compensator, the off-shell constraint on $H^{i j}$, eq. (7.1), implies the following relations:

$$
\begin{aligned}
X_{\hat{a} \hat{b}}+N_{\hat{a} \hat{b}} & =\frac{\mathrm{i}}{4}\left(\Sigma_{\hat{a} \hat{b}}\right)^{\hat{\alpha} \hat{\beta}} H^{\frac{1}{2}} \mathcal{D}_{\hat{\alpha}}^{k} \mathcal{D}_{\hat{\beta} k} H^{-\frac{1}{2}} ; \\
S^{(i}{ }_{k} H^{j) k} & =-\frac{\mathrm{i}}{48 H^{2}} H^{(i}{ }_{k}\left(\mathcal{D}^{\hat{\alpha} j)} \mathcal{D}_{\hat{\alpha}}^{k} H^{2}-2\left(\mathcal{D}^{\hat{\alpha} j)} H\right) \mathcal{D}_{\hat{\alpha}}^{k} H\right) ; \\
C_{\hat{a} k}^{(i} H^{j) k} & =-\mathcal{D}_{\hat{a}} H^{i j}-\frac{\mathrm{i}}{16 H^{2}}\left(\Gamma_{\hat{a}}\right)^{\hat{\alpha} \hat{\beta}} H^{(i}{ }_{k}\left(\mathcal{D}_{\hat{\alpha}}^{j)} \mathcal{D}_{\hat{\beta}}^{k} H^{2}-2\left(\mathcal{D}_{\hat{\alpha}}^{j)} H\right) \mathcal{D}_{\hat{\beta}}^{k} H\right) .
\end{aligned}
$$

The first relation completely determines $X_{\hat{a} \hat{b}}+N_{\hat{a} \hat{b}}$ in terms of $H^{i j}$. In the super-Weyl gauge $H=1$, the relations (8.2) reduce to (7.5).

\subsection{Supersymmetric backgrounds}

Looking at the relations (8.1) and (8.2), it appears that the super-Weyl gauge $H=1$ is simpler to deal with. This gauge condition and its implications, worked out in section 7 , will be used in the remainder of this section. We have to analyse the implications of the supersymmetry invariance of $W$,

$$
\xi^{\hat{A}} \mathcal{D}_{\hat{A}} W=0 .
$$

As before, we are interested in purely bosonic backgrounds, and thus we require

$$
\mathcal{D}_{\hat{\alpha}}^{i} W \mid=0 .
$$

Demanding this condition to be supersymmetric, $\delta\left(\mathcal{D}_{\hat{\alpha}}^{i} W\right) \mid=0$, gives

$$
\begin{aligned}
\epsilon_{i}^{\hat{\alpha}}\left[\varepsilon_{\hat{\alpha} \hat{\beta}} \varepsilon^{\hat{\gamma} \hat{\delta}} \mathcal{D}_{\hat{\gamma}}^{(i} \mathcal{D}_{\hat{\delta}}^{j)}+\left(\Gamma^{\hat{a}}\right)_{\hat{\alpha} \hat{\beta}}\left(\Gamma_{\hat{a}}\right)^{\hat{\gamma} \hat{\delta}}\left(\mathcal{D}_{\hat{\gamma}}^{(i} \mathcal{D}_{\hat{\delta}}^{j)}-\mathrm{i} \varepsilon^{i j} \mathcal{D}_{\hat{\alpha} \hat{\beta}}\right)\right. \\
+\varepsilon^{i j}\left(\Sigma^{\hat{a} \hat{b}}\right)_{\hat{\alpha} \hat{\beta}}\left(\Sigma_{\hat{a} \hat{b}} \hat{\gamma} \hat{\delta} \hat{\mathcal{D}} \mathcal{D}_{\hat{\gamma}}^{k} \mathcal{D}_{\hat{\delta} k}\right] W \mid=0 .
\end{aligned}
$$

This is equivalent to

$$
\left[y^{i j} \mathbb{1}+2 \mathrm{w} c_{\hat{a}}^{i j} \Gamma^{\hat{a}}-4 \varepsilon^{i j} \Gamma^{\hat{a}} \mathbf{D}_{\hat{a}} \mathrm{w}+4 \varepsilon^{i j}\left(f_{\hat{a} \hat{b}}-\mathrm{w} x_{\hat{a} \hat{b}}\right) \Sigma^{\hat{a} \hat{b}}\right] \epsilon_{j}=0,
$$


where we have introduced the component fields

$$
\mathrm{w}:=W\left|, \quad y^{i j}:=\mathrm{i} \mathcal{D}^{\hat{\gamma}(i} \mathcal{D}_{\hat{\gamma}}^{j)} W\right| .
$$

By construction, the scalar $\mathrm{w}$ is nowhere vanishing. Eq. (8.6) is the additional condition on any supersymmetric background, which comes from the vector compensator. The other conditions are given in subsection 7.1.

\subsection{Supersymmetric backgrounds with eight supercharges}

In the case of maximally supersymmetric backgrounds, equation (8.6) is solved by

$$
\mathrm{w}=\text { const }, \quad y^{i j}=0, \quad c_{\hat{a}}^{i j}=0, \quad x_{\hat{a} \hat{b}}=\frac{1}{\mathrm{w}} f_{\hat{a} \hat{b}} .
$$

It should be kept in mind that the two-form $f:=\frac{1}{2} f_{\hat{a}} e^{\hat{a}} e^{\hat{b}}$ is a U(1) field strength, and hence it is closed, $\mathrm{d} f=0$.

Since we consider the maximally supersymmetric backgrounds, it follows from eq. (8.4) that

$$
\mathcal{D}_{\hat{\alpha}}^{i} W=0 \quad \Longrightarrow \quad W=\text { const } .
$$

The first and second conditions in (8.8) are corollaries of this result. From (8.1a) we also deduce

$$
C_{\hat{a}}^{i j}=0
$$

We can now use the results of section 5 to describe maximally supersymmetric backgrounds in off-shell supergravity. Note that in our case $c_{\hat{a}}^{i j}=0$ and $n_{\hat{a} \hat{b}}=-x_{\hat{a} \hat{b}}$.

\subsubsection{The case $s \neq 0$}

When the scalar $s$ is nonzero, all conclusions of subsection 7.2 hold. In particular, the spacetime has $\mathrm{AdS}_{5}$ geometry.

\subsubsection{The case $s=0$}

It remains to consider the case $s=0$. Then $s^{i j}=0$ and $c_{\hat{a}}^{i j}=0$, and the geometry is formulated in terms of a single two-form $x=\frac{1}{2} x_{\hat{a} \hat{b}} e^{\hat{a}} e^{\hat{b}}$ such that

$$
\mathbf{D}_{\hat{a}} x_{\hat{b} \hat{c}}=\frac{1}{8} \eta_{\hat{a}[\hat{b}} \varepsilon_{\hat{c}]} \hat{d} \hat{e} \hat{f} \hat{g} x^{\hat{d} \hat{e}} x^{\hat{f} \hat{g}} .
$$

This equation implies that the two-form $x$ is closed, $\mathrm{d} x=0$, which is consistent with the relation $x_{\hat{a} \hat{b}}=(1 / \mathrm{w}) f_{\hat{a} \hat{b}}$. 


\section{Supersymmetric solutions in Poincaré and anti-de Sitter supergravities}

In sections 6 and 7, we studied the restrictions on supersymmetric backgrounds that originate due to the presence of a single conformal compensator. In section 8 we considered the off-shell supergravity formulation obtained by coupling the Weyl multiplet to two compensators: (i) the vector multiplet; and (ii) the $\mathcal{O}(2)$ multiplet. It was demonstrated that the presence of a second compensator leads to additional restrictions on supersymmetric backgrounds. Now we turn to analysing supersymmetric solutions in this supergravity theory, with or without a cosmological term. Our analysis will be restricted to the case of on-shell supergravity backgrounds.

It may be shown that the supergravity equations of motion ${ }^{10}$ are

$$
\begin{aligned}
H-W^{3} & =0, \\
\mathbb{H}^{i j}+\chi H^{i j} & =0, \\
\mathbb{W}+3 \chi W & =0,
\end{aligned}
$$

with $\chi$ the cosmological constant. Here $\mathbb{H}^{i j}$ is the composite $\mathcal{O}(2)$ multiplet (6.12), and $\mathbb{W}$ is a composite vector multiplet constructed out of the $\mathcal{O}(2)$ compensator. The latter is defined by

$$
\mathbb{W}=\overline{\mathbb{W}}=\frac{\mathrm{i}}{4} H\left(\mathcal{D}^{i j}+12 \mathrm{i} S^{i j}\right)\left(\frac{H_{i j}}{H^{2}}\right)
$$

and obeys the Bianchi identity

$$
\mathcal{D}_{\hat{\alpha}}^{(i} \mathcal{D}_{\hat{\beta}}^{j)} \mathbb{W}-\frac{1}{4} \varepsilon_{\hat{\alpha} \hat{\beta}} \mathcal{D}^{\hat{\gamma}(i} \mathcal{D}_{\hat{\gamma}}^{j)} \mathbb{W}=\frac{i}{2} C_{\hat{\alpha} \hat{\beta}}^{i j} \mathbb{W}
$$

Its super-Weyl transformation law is

$$
\delta_{\sigma} \mathbb{W}=\sigma \mathbb{W}
$$

Let us comment on the equations of motion (9.1). The supergravity theory is described in terms of three interacting multiplets: (i) the Weyl multiplet; (ii) the vector multiplet; and (iii) the $\mathcal{O}(2)$ multiplet. It may be shown that, modulo gauge freedom, the Weyl multiplet is described by a single unconstrained real prepotential $G .{ }^{11}$ The equation (9.1a) is obtained by varying the supergravity action with respect to $G$. The meaning of (9.1a) is that the supercurrent of pure supergravity is equal to zero.

In general, given a super-Weyl invariant theory of dynamical (matter) superfields $\varphi^{i}$ coupled to the Weyl multiplet, the supercurrent of this theory is a real scalar superfield defined by

$$
\mathcal{T}=\frac{\Delta}{\Delta G} S[\varphi],
$$

\footnotetext{
${ }^{10}$ Similar equations of motion occur in $4 \mathrm{D} \mathcal{N}=2$ (gauged) supergravity [56, 57].

${ }^{11}$ This can be done in complete analogy with the case of $4 \mathrm{D} \mathcal{N}=2$ supergravity [58].
} 
where $\Delta / \Delta G$ denotes a covariantized variational derivative with respect to $G$. The supercurrent turns out to obey the conservation equation ${ }^{12}$

$$
\left(\mathcal{D}^{i j}+12 \mathrm{i} S^{i j}\right) \mathcal{T}=0
$$

provided the dynamical superfields obey their equations of motion, $\delta S[\varphi] / \delta \varphi^{i}=0$. The super-Weyl transformation law of $\mathcal{T}$ is

$$
\delta_{\sigma} \mathcal{T}=3 \sigma \mathcal{T},
$$

which makes the equation (9.6) super-Weyl invariant. It is an instructive exercise to prove that the left-hand side of (9.1a) obeys the constraint

$$
\left(\mathcal{D}^{i j}+12 \mathrm{i} S^{i j}\right)\left(H-W^{3}\right)=0
$$

provided the equations (9.1b) and (9.1c) hold.

The equations of motion (9.1b) and (9.1c) correspond to the vector and $\mathcal{O}(2)$ compensators, respectively. The derivation of these equations will be given elsewhere.

Note that we can always choose the super-Weyl gauge (7.3),

$$
H=1 \text {. }
$$

As shown in section 7 , this gauge condition implies

$$
\mathcal{D}_{\hat{\alpha}}^{i} H^{j k}=0, \quad S^{i j}=S H^{i j},
$$

for some scalar superfield $S$. Due to the equation of motion (9.1a), the field strength $W$ also becomes constant,

$$
W=1
$$

Moreover, both eqs. (9.1b) and (9.1c) become equivalent to

$$
S=\frac{1}{2} \chi
$$

Since $W=1$ and $H=1$ on the mass shell, it holds that

$$
C_{\hat{a}}^{k l}=0, \quad X_{\hat{a} \hat{b}}=F_{\hat{a} \hat{b}}=-N_{\hat{a} \hat{b}} .
$$

Due to $(7.5 \mathrm{c}), H^{k l}$ is actually covariantly constant,

$$
\mathcal{D}_{\hat{A}} H^{k l}=0,
$$

and therefore the $\mathrm{SU}(2)$ curvature factorizes,

$$
R_{\hat{A} \hat{B}}^{k l}=R_{\hat{A} \hat{B}} H^{k l},
$$

\footnotetext{
${ }^{12}$ The supercurrent multiplet in $5 \mathrm{D} \mathcal{N}=1$ Poincaré supersymmetry was introduced by Howe and Lindström [14].
} 
for a closed super two-form $R_{\hat{A} \hat{B}}$ given by

$$
R_{\hat{\alpha} \hat{\beta}}^{i j}=\frac{3 \mathrm{i}}{2} \chi \varepsilon_{\hat{\alpha} \hat{\beta}} \varepsilon^{i j}, \quad R_{\hat{a} \hat{\beta}}^{j}=0, \quad R_{\hat{a} \hat{b}}=-\frac{3}{4} \chi F_{\hat{a} \hat{b}} .
$$

This super two-form proves to be proportional to the $\mathrm{U}(1)$ field strength $F_{\hat{A} \hat{B}}$, eq. (6.3),

$$
R_{\hat{A} \hat{B}}=-\frac{3}{4} \chi F_{\hat{A} \hat{B}} .
$$

Now the local SU(2) symmetry may be used to choose the corresponding connection in the form $\Phi_{\hat{A}}^{k l}=\Phi_{\hat{A}} H^{k l}$. As a result, the $\mathrm{SU}(2)$ group reduces to a U(1) subgroup generated by $\mathcal{J}:=-\mathrm{i} H^{k l} J_{k l}$. Due to $(9.17)$, we may identify (up to a factor) $\Phi_{\hat{A}}$ with the $\mathrm{U}(1)$ connection $\mathcal{V}_{\hat{A}}$ in $(6.1)$.

For on-shell supergravity under consideration, we are interested in backgrounds that possess some rigid supersymmetry. Using the gauge conditions described, the Killing spinor equation (6.8) turns into

$$
\mathbf{D}_{\hat{a}} \epsilon^{k}=\frac{1}{4} \chi \Gamma_{\hat{a}} h^{k}{ }_{l} \epsilon^{l}+\frac{1}{8} \delta_{l}^{k} f_{\hat{b} \hat{c}}\left(\varepsilon_{\hat{a}}^{\hat{b} \hat{c}} \hat{d e} \Sigma_{\hat{d} \hat{e}}+4 \delta_{\hat{a}}^{\hat{b}} \Gamma^{\hat{c}}\right) \epsilon^{l} .
$$

The Killing spinor equation (9.18) coincides with the one derived in [59]. In the case of Poincaré supergravity, $\chi=0$, it reduces to the Killing spinor equation given in [51]. The supersymmetric backgrounds for on-shell simple Poincaré and anti-de Sitter supergravity theories in five dimensions have been studied in detail in [51] and [59], respectively. There is no need to repeat here the analysis given there.

In the case of anti-de Sitter supergravity, $\chi \neq 0$, the isovector $s^{i j}$ is non-zero, $s^{i j}=$ $\frac{1}{2} \chi h^{i j}$. Then our earlier analysis implies that $\mathrm{AdS}_{5}$ is the only maximally supersymmetric solution. This agrees with the conclusions of [59].

\section{Concluding comments}

In this paper we have developed the formalism to construct off-shell supersymmetric backgrounds within the superspace formulation for 5D conformal supergravity [3]. For those superspace backgrounds which obey the equations of motion for Poincaré or antide Sitter supergravity, we have naturally reproduced the supersymmetric solutions constructed in $[51,59]$.

Although we presented a number of supersymmetric backgrounds, a classification of such semi-Riemannian spaces was not our goal. Given a semi-Riemannian space that admits at least one rigid supersymmetry, our ultimate aim was to embed it in a curved background superspace such that its geometry is of the type described in section 2. After that it becomes trivial to generate rigid supersymmetric theories on this space by making use of the offshell supergravity-matter systems presented in [1-3]. In this sense, the curved superspace approach is much more powerful than the Noether procedure advocated, e.g., in [27].

To illustrate the power of the curved superspace approach at generating rigid supersymmetric theories, it suffices to consider the example of 5D anti-de Sitter space. Eight years ago, two of us [18] constructed the most general off-shell supersymmetric nonlinear 
$\sigma$-models in 5D $\mathcal{N}=1$ AdS superspace formulated in terms of covariant weight-zero polar hypermultiplets. A year later, the construction of [18] was extended to the case of 4D $\mathcal{N}=2$ AdS supersymmetry [20]. However, since the $\sigma$-models proposed in $[18,20]$ made use of off-shell supermultiplets with infinitely many auxiliary fields, which have never been dealt with in the framework of superconformal tensor calculus, these theories remained largely unnoticed. In 2011, two separate developments took place. The most general nonlinear $\sigma$-models with $4 \mathrm{D} \mathcal{N}=2 \mathrm{AdS}$ and $5 \mathrm{D} \mathcal{N}=1 \mathrm{AdS}$ supersymmetries were constructed in $[21,22]$ and $[60,61]$, respectively, in terms of $4 \mathrm{D} \mathcal{N}=1$ chiral superfields. ${ }^{13}$ The common feature of the $4 \mathrm{D} \mathcal{N}=2$ and $5 \mathrm{D} \mathcal{N}=1 \mathrm{AdS}$ supersymmetries is that the $\sigma$-model target spaces are those hyperkähler manifolds which possess a Killing vector field generating an $\mathrm{SO}(2)$ group of rotations on the two-sphere of complex structures. ${ }^{14}$ Not all hyperkähler manifolds possess such an $\mathrm{SO}(2)$ isometry group. This clearly differs from the $4 \mathrm{D} \mathcal{N}=2$ or $5 \mathrm{D} \mathcal{N}=1$ Poincaré supersymmetries where arbitrary hyperkähler manifolds can originate as target spaces of supersymmetric $\sigma$-models [63, 64]. In 2012, ref. [23] established the one-to-one correspondence between the two types of $\mathcal{N}=2$ supersymmetric $\sigma$-models in $\mathrm{AdS}_{4}$ : the off-shell [20] and the on-shell [21,22] ones. Similar considerations may be used to establish a one-to-one correspondence between the $\mathcal{N}=1$ supersymmetric $\sigma$-models in $\mathrm{AdS}_{5}$ constructed in [18] and [60].

The off-shell supersymmetric $\sigma$-models with eight supercharges in $\mathrm{AdS}_{4}$ [20] and $\mathrm{AdS}_{5}$ [18] are constant-curvature deformations of the family of $\mathcal{N}=2$ rigid supersymmetric $\sigma$-models in $\mathbb{R}^{3,1}$ introduced in [65] and studied in [66, 67] (see also [48] for the 5D $\mathcal{N}=1$ extension). ${ }^{15}$ The target space $\mathcal{M}$ of such a nonlinear $\sigma$-model was shown in [65-67] to be an open domain of the zero section of the cotangent bundle $T^{*} \mathcal{X}$ of a real analytic Kähler manifold $\mathcal{X}$ (the off-shell $\sigma$-model action [65] is constructed in terms of the Kähler potential $K(\Phi, \bar{\Phi})$ of $\mathcal{X})$. Since the target spaces of any $4 \mathrm{D} \mathcal{N}=2$ rigid supersymmetric $\sigma$-models are hyperkähler [63], $\mathcal{M}$ is a hyperkähler manifold, for any real analytic Kähler manifold $\mathcal{X}$. Thus the superspace construction of [65-67] provided a proof that there exists a hyperkähler structure on an open domain of the zero section of the cotangent bundle $T^{*} \mathcal{X}$ of a real analytic Kähler manifold $\mathcal{X}$. This proof is much simpler than the ones given in the mathematical literature [68-70] and appeared two years earlier than [70]. ${ }^{16}$ For any real analytic Kähler manifold $\mathcal{X}$, the off-shell $\sigma$-model action of [65-67] possesses a $\mathrm{U}(1)$ rigid symmetry, which manifests in a certain $\mathrm{U}(1)$ isometry of the hyperkähler space $T^{*} \mathcal{X}$. This $\mathrm{U}(1)$ isometry acts by scalar multiplication in the fibres and rotates the complex structures. This $\mathrm{U}(1)$ isometry group of $T^{*} \mathcal{X}$ plays an important role in [68-70].

\footnotetext{
${ }^{13}$ The component formulation of the $5 \mathrm{D} \mathcal{N}=1$ supersymmetric $\sigma$-models constructed in [60] was given in $[61]$.

${ }^{14}$ Such hyperkähler manifolds were first described in [62].

${ }^{15}$ The supersymmetric $\sigma$-models introduced in [65] form a special subfamily in the general family of polar multiplet $\sigma$-models pioneered by Lindström and Roček [16, 17].

${ }^{16}$ One of the authors of [66] (SMK) was informed about Kaledin's work [68, 69] only after his talk, which was given at the 32nd International Symposium Ahrenshoop on the Theory of Elementary Particles (1-5 September 1998, Buckow, Germany) and in which the results of [66] were announced. Ref. [67] is a written version of the talk given.
} 
In the case of maximally supersymmetric backgrounds considered in subsection 5.2 , it is not difficult to construct a family of rigid supersymmetric $\sigma$-models as a generalization of the locally supersymmetric off-shell nonlinear $\sigma$-models given in [1-3]. The dynamical variables of such a theory are a set of interacting covariantly arctic weight-zero multiplets $\Upsilon^{I}$ and their smile-conjugates $\breve{\Upsilon}^{\bar{I}}$, and the dynamics is described by a projective-superspace Lagrangian of the form

$$
\mathcal{L}^{++}=C^{++} K(\Upsilon, \breve{\Upsilon}), \quad C^{++}=C^{i j} u_{i}^{+} u_{j}^{+}
$$

where $K\left(\Phi^{I}, \bar{\Phi}^{\bar{J}}\right)$ is the Kähler potential of a real analytic Kähler manifold $\mathcal{M}$, and $u_{i}^{+}$are homogeneous complex coordinates for $\mathbb{C} P^{1}$. The supersymmetric action constructed from $\mathcal{L}^{++}$proves to be invariant under Kähler transformations of the form

$$
K(\Upsilon, \breve{\Upsilon}) \rightarrow K(\Upsilon, \breve{\Upsilon})+\Lambda(\Upsilon)+\bar{\Lambda}(\breve{\Upsilon})
$$

with $\Lambda\left(\Phi^{I}\right)$ a holomorphic function. It is of interest to understand the target-space geometry of such nonlinear $\sigma$-models, in particular its dependence on the 5 -vector parameter $C^{\hat{a}}$ of the curved superspace under consideration.

It appears that only superconformal $\sigma$-sigma models can be consistently defined in the case of those maximally supersymmetric backgrounds in subsection 5.3 that are characterized by the condition $X_{\hat{a} \hat{b}}+N_{\hat{a} \hat{b}} \neq 0$, because the holonomy group of the superspace (5.46) then includes the $R$-symmetry group SU(2). However, if $X_{\hat{a} \hat{b}}+N_{\hat{a} \hat{b}}=0$, the $\mathrm{SU}(2)$ curvature is identically zero.

In our discussion of 5D supersymmetric backgrounds, the bosonic conditions (4.1) were postulated. Actually such conditions naturally originate as consistency requirements for the existence of rigid supersymmetry transformations. Indeed, let $\mathcal{T}$ be any bosonic component of the superspace torsion and curvature tensors in (2.7), which correspond to a supersymmetric background. The variation of $\mathcal{T}$ under a rigid supersymmetry transformation must vanish, and hence

$$
0=\epsilon_{i}^{\hat{\alpha}} \mathcal{D}_{\hat{\alpha}}^{i} \mathcal{T} \mid
$$

where we have made use of the conditions $K^{\hat{a} \hat{b}}\left|=0, K^{i j}\right|=0$ and $\sigma[\xi]=0$. For this to hold, it suffices to require the spinor component $\mathcal{D}_{\hat{\alpha}}^{i} \mathcal{T} \mid$ to vanish, $\mathcal{D}_{\hat{\alpha}}^{i} \mathcal{T} \mid=0$. On the other hand, if we are only interested in those backgrounds that possess conformal supersymmetries, it is not necessary to impose the bosonic conditions (4.1). To see this, let us start from a purely bosonic background possessing a conformal supersymmetry and then introduce a conformally related superspace defined by (3.14). For the latter superspace, the requirements (4.10) still hold, but some of the conditions $K^{\hat{a} \hat{b}}\left|=0, K^{i j}\right|=0$ and $\sigma \mid=0$ are no longer true. Moreover, some fermionic components of the superspace torsion and curvature tensors may be non-zero.

Recently, there have appeared two publications devoted to supersymmetric backgrounds for $5 \mathrm{D} \mathcal{N}=1$ supergravity with Euclidean signature [71, 72]. Our conformal Killing equation (4.23) is analogous to those given in [71, 72]. 


\section{Acknowledgments}

We are grateful to Daniel Butter for valuable suggestions and comments on the manuscript. This work is supported in part by the Australian Research Council projects DP1096372, DE120101498 and DP140103925.

\section{A (Conformal) isometries in curved space}

In this appendix we recall how the problem of computing the (conformal) isometries of a curved spacetime is addressed within the Weyl-invariant formulation for gravity [73]. Our presentation follows [74].

We start by recalling three known approaches to the description of gravity in $d$ dimensions: (i) metric formulation; (ii) vielbein formulation; and (iii) Weyl-invariant formulation. In the standard metric approach, the gauge field is a metric tensor $g_{m n}(x)=g_{n m}(x)$ constrained to be nonsingular, $g:=\operatorname{det}\left(g_{m n}\right) \neq 0$. The gauge transformation is

$$
\delta g_{m n}=\nabla_{m} \xi_{n}+\nabla_{n} \xi_{m},
$$

with the gauge parameter $\xi=\xi^{m}(x) \partial_{m}$ being a vector field generating an infinitesimal diffeomorphism.

In the vielbein formulation, the gauge field is a vielbein $e_{m}{ }^{a}(x)$ that constitutes a basis in the tangent space at $x$, for any spacetime point $x, e:=\operatorname{det}\left(e_{m}{ }^{a}\right) \neq 0$. The metric becomes a composite field defined by $g_{m n}=e_{m}{ }^{a} e_{n}{ }^{b} \eta_{a b}$. The gauge group is now larger than in the metric approach. It includes general coordinate and local Lorentz transformations,

$$
\delta \nabla_{a}=\left[\xi^{b} \nabla_{b}+\frac{1}{2} K^{b c} M_{b c}, \nabla_{a}\right],
$$

with the gauge parameters $\xi^{a}(x)=\xi^{m}(x) e_{m}{ }^{a}(x)$ and $K^{a b}(x)=-K^{b a}(x)$ being completely arbitrary. The gauge transformation makes use of the torsion-free covariant derivatives

$$
\nabla_{a}=e_{a}^{m} \partial_{m}+\frac{1}{2} \omega_{a}^{b c} M_{b c}, \quad\left[\nabla_{a}, \nabla_{b}\right]=\frac{1}{2} R_{a b}{ }^{c d} M_{c d} .
$$

Here $M_{b c}=-M_{c b}$ denotes the Lorentz generators, $e_{a}{ }^{m}$ the inverse vielbein, $e_{a}{ }^{m} e_{m}{ }^{b}=\delta_{a}^{b}$, and $\omega_{a}^{b c}$ the torsion-free Lorentz connection.

As is well known, the torsion-free constraint

$$
T_{a b}{ }^{c}=0 \quad \Longleftrightarrow \quad\left[\nabla_{a}, \nabla_{b}\right] \equiv T_{a b}{ }^{c} \nabla_{c}+\frac{1}{2} R_{a b}{ }^{c d} M_{c d}=\frac{1}{2} R_{a b}{ }^{c d} M_{c d}
$$

is invariant under Weyl (local scale) transformations

$$
\nabla_{a} \rightarrow \nabla_{a}^{\prime}=\mathrm{e}^{\sigma}\left(\nabla_{a}+\left(\nabla^{b} \sigma\right) M_{b a}\right)
$$

with the parameter $\sigma(x)$ being completely arbitrary. This transformation is induced by that of the gravitational field

$$
e_{a}^{m} \rightarrow \mathrm{e}^{\sigma} e_{a}{ }^{m} \quad \Longrightarrow \quad g_{m n} \rightarrow \mathrm{e}^{-2 \sigma} g_{m n} .
$$


Most field theories in curved space do not possess Weyl invariance. In particular, the pure gravity action with a cosmological term

$$
S=\frac{1}{2 \kappa^{2}} \int \mathrm{d}^{d} x e R-\frac{\Lambda}{\kappa^{2}} \int \mathrm{d}^{d} x e
$$

is not invariant under the Weyl transformations (A.5).

Weyl-invariant matter theories are curved-space extensions of ordinary conformally invariant theories. As an example, consider the model for a scalar field $\varphi$ with action

$$
S=-\frac{1}{2} \int \mathrm{d}^{d} x e\left\{\nabla^{a} \varphi \nabla_{a} \varphi+\frac{1}{4} \frac{d-2}{d-1} R \varphi^{2}+\lambda \varphi^{2 d /(d-2)}\right\}
$$

with $R$ the scalar curvature and $\lambda$ a coupling constant. The action is Weyl invariant ${ }^{17}$ provided $\varphi$ transforms as

$$
\varphi \rightarrow \varphi^{\prime}=\mathrm{e}^{\frac{1}{2}(d-2) \sigma} \varphi
$$

In the Weyl-invariant formulation for gravity, the gravitational field is described in terms of two gauge fields. One of them is the vielbein $e_{m}{ }^{a}(x)$ and the other is a conformal compensator $\varphi(x)$ with the Weyl transformation law (A.9). Unlike the matter model (A.8), the compensator is constrained to be nowhere vanishing, $\varphi \neq 0$. The gravity gauge group is defined to include the general coordinate, local Lorentz and Weyl transformations

$$
\begin{aligned}
\delta \nabla_{a} & =\left[\xi^{b} \nabla_{b}+\frac{1}{2} K^{b c} M_{b c}, \nabla_{a}\right]+\sigma \nabla_{a}+\left(\nabla^{b} \sigma\right) M_{b a} \equiv\left(\delta_{\mathcal{K}}+\delta_{\sigma}\right) \nabla_{a}, \\
\delta \varphi & =\xi^{b} \nabla_{b} \varphi+\frac{1}{2}(d-2) \sigma \varphi \equiv\left(\delta_{\mathcal{K}}+\delta_{\sigma}\right) \varphi
\end{aligned}
$$

where we have denoted $\mathcal{K}:=\xi^{b} \nabla_{b}+\frac{1}{2} K^{b c} M_{b c}$. In this approach, any dynamical system is required to be invariant under the general coordinate, local Lorentz and Weyl transformations. In particular, the Weyl-invariant gravity action is

$$
S=\frac{1}{2} \int \mathrm{d}^{d} x e\left\{\nabla^{a} \varphi \nabla_{a} \varphi+\frac{1}{4} \frac{d-2}{d-1} R \varphi^{2}+\lambda \varphi^{2 d /(d-2)}\right\} .
$$

Applying a finite Weyl transformation allows us to choose a gauge

$$
\varphi=\frac{1}{2 \kappa} \sqrt{\frac{d-1}{d-2}}
$$

in which the action turns into (A.7).

A vector field $\xi=\xi^{m} \partial_{m}=\xi^{a} e_{a}$, with $e_{a}:=e_{a}{ }^{m} \partial_{m}$, is conformal Killing if there exist local Lorentz $K^{b c}[\xi]$ and Weyl $\sigma[\xi]$ parameters such that

$$
\left[\xi^{b} \nabla_{b}+\frac{1}{2} K^{b c}[\xi] M_{b c}, \nabla_{a}\right]+\sigma[\xi] \nabla_{a}+\left(\nabla^{b} \sigma[\xi]\right) M_{b a}=0 .
$$

\footnotetext{
${ }^{17}$ The Weyl transformation law of $R$ is $R \rightarrow \mathrm{e}^{2 \sigma}\left\{R+2(d-1) \nabla^{a} \nabla_{a} \sigma-(d-2)(d-1)\left(\nabla^{a} \sigma\right) \nabla_{a} \sigma\right\}$.
} 
A short calculation gives

$$
K^{b c}[\xi]=\frac{1}{2}\left(\nabla^{b} \xi^{c}-\nabla^{c} \xi^{b}\right), \quad \sigma[\xi]=\frac{1}{d} \nabla_{b} \xi^{b}
$$

as well as the conformal Killing equation

$$
\nabla^{a} \xi^{b}+\nabla^{b} \xi^{a}=2 \eta^{a b} \sigma[\xi] .
$$

The set of all conformal Killing vector fields of a given spacetime is a finite-dimensional Lie algebra with respect to the standard Lie bracket for vector fields. It is the conformal algebra of the spacetime.

Two spacetimes $\left(\nabla_{a}, \varphi\right)$ and $\left(\widetilde{\nabla}_{a}, \widetilde{\varphi}\right)$ are said to be conformal if their covariant derivatives are related to each other as follows:

$$
\widetilde{\nabla}_{a}=\mathrm{e}^{\rho}\left(\nabla_{a}+\left(\nabla^{b} \rho\right) M_{b a}\right), \quad \widetilde{\varphi}=\mathrm{e}^{\frac{1}{2}(d-2) \rho} \varphi,
$$

for some $\rho$. These spacetimes have the same conformal Killing vector fields $\xi=\xi^{a} e_{a}=\tilde{\xi}^{a} \tilde{e}_{a}$. The parameters $K^{c d}[\tilde{\xi}]$ and $\sigma[\tilde{\xi}]$ are related to $K^{c d}[\xi]$ and $\sigma[\xi]$ as follows:

$$
\begin{aligned}
\mathcal{K}[\tilde{\xi}] & :=\tilde{\xi}^{b} \widetilde{\nabla}_{b}+\frac{1}{2} K^{c d}[\tilde{\xi}] M_{c d}=\mathcal{K}[\xi], \\
\sigma[\tilde{\xi}] & =\sigma[\xi]-\xi \rho .
\end{aligned}
$$

A vector field $\xi=\xi^{m} \partial_{m}=\xi^{a} e_{a}$, with $e_{a}:=e_{a}{ }^{m} \partial_{m}$, is Killing if there exist local Lorentz $K^{b c}[\xi]$ and Weyl $\sigma[\xi]$ parameters such that

$$
\begin{aligned}
{\left[\xi^{b} \nabla_{b}+\frac{1}{2} K^{b c}[\xi] M_{b c}, \nabla_{a}\right]+\sigma[\xi] \nabla_{a}+\left(\nabla^{b} \sigma[\xi]\right) M_{b a} } & =0 \\
\xi \varphi+\frac{1}{2}(d-2) \sigma[\xi] \varphi & =0 .
\end{aligned}
$$

The set of all conformal Killing vector fields of a given spacetime is a finite-dimensional Lie algebra. By construction, it is a subalgebra of the conformal algebra of the spacetime. The Killing equations (A.19) are Weyl invariant in the following sense. Given a conformally related spacetime $\left(\widetilde{\nabla}_{a}, \widetilde{\varphi}\right)$ defined by eq. (A.16), the Killing equations (A.19) have the same functional form when rewritten in terms of $\left(\widetilde{\nabla}_{a}, \widetilde{\varphi}\right)$. In particular,

$$
\xi \widetilde{\varphi}+\frac{1}{2}(d-2) \sigma[\tilde{\xi}] \widetilde{\varphi}=0
$$

Due to Weyl invariance, we can work with a conformally related spacetime such that

$$
\varphi=1 \text {. }
$$

Then for $d>2$ the Killing equations turn into

$$
\left[\xi^{b} \nabla_{b}+\frac{1}{2} K^{b c}[\xi] M_{b c}, \nabla_{a}\right]=0, \quad \sigma[\xi]=0 .
$$

This is equivalent to the standard Killing equation

$$
\nabla^{a} \xi^{b}+\nabla^{b} \xi^{a}=0
$$




\section{B Conformal Killing spinors and bilinears}

The famous classification of supersymmetric solutions [51, 59] in 5D $\mathcal{N}=1$ Poincaré and anti-de Sitter supergravity theories was based on the use of the algebraic and differential properties of bilinears constructed from a Killing spinor. In this appendix we study the properties of such bilinears associated with (conformal) Killing spinors in off-shell supergravity.

Given a commuting spinor $\epsilon_{\hat{\alpha}}^{i}$, we may construct the following real bilinears:

$$
\begin{aligned}
F & :=\epsilon_{\hat{\gamma}}^{k} \epsilon_{k}^{\hat{\gamma}}, \\
V_{\hat{a}} & :=\left(\Gamma_{\hat{a}}\right)^{\hat{\alpha} \hat{\beta}} \epsilon_{\hat{\alpha}}^{k} \epsilon_{\hat{\beta} k}, \\
G_{\hat{a} \hat{b}}{ }^{i j} & :=-\left(\Sigma_{\hat{a} \hat{b}}\right)^{\hat{\alpha} \hat{\beta}} \epsilon_{\hat{\alpha}}^{(i} \epsilon_{\hat{\beta}}^{j)}=G_{[\hat{a} \hat{b}]}{ }^{(i j)} .
\end{aligned}
$$

It is straightforward to show that the above bilinears satisfy the following algebraic identities:

$$
\begin{aligned}
V^{\hat{a}} V_{\hat{a}} & =-F^{2}, \\
\varepsilon^{\hat{a} \hat{b} \hat{c} \hat{d} \hat{e}} G_{\hat{b} \hat{c}}^{i j} G_{\hat{d} \hat{e} k l} & =-\delta_{(k}^{i} \delta_{l)}^{j} V^{\hat{a}} F, \\
V^{\hat{a}} G_{\hat{a} \hat{b}}^{i j} & =0, \\
\varepsilon^{\hat{a} \hat{b} \hat{c} \hat{d} \hat{e}} V_{\hat{c}} G_{\hat{d} \hat{e}}^{i j} & =2 G^{\hat{a} \hat{b} i j} F, \\
G_{\hat{a}}{ }^{\hat{c} i j} G_{\hat{c} \hat{b}}^{k l} & =\frac{1}{8} \varepsilon^{k(i} \varepsilon^{j) l}\left(\eta_{\hat{a} \hat{b}} F^{2}+V_{\hat{a}} V_{\hat{b}}\right)+\frac{1}{4} \varepsilon^{k(i} F G_{\hat{a} \hat{b}}^{j) l}+\frac{1}{4} \varepsilon^{l(i} F G_{\hat{a} \hat{b}}^{j) k}, \\
V_{\hat{\alpha} \hat{\beta}} \epsilon^{\hat{\beta} j} & =F \epsilon_{\hat{\alpha}}^{j}, \\
G_{\hat{\alpha} \hat{\beta}}{ }^{i j} \epsilon^{\hat{\beta} k} & =-\frac{1}{2} \varepsilon^{k(i} F \epsilon_{\hat{\alpha}}^{j)},
\end{aligned}
$$

where

$$
V_{\hat{\alpha} \hat{\beta}}=\left(\Gamma^{\hat{a}}\right)_{\hat{\alpha} \hat{\beta}} V_{\hat{a}}, \quad G_{\hat{\alpha} \hat{\beta}}^{i j}=\frac{1}{2}\left(\Sigma^{\hat{a} \hat{b}}\right)_{\hat{\alpha} \hat{\beta}} G_{\hat{a} \hat{b}}^{i j} .
$$

Eq. (B.2a) tells us that the five-vector $V^{\hat{a}}$ is time-like or null.

Let $\epsilon_{\hat{\alpha}}^{i}$ be a conformal Killing spinor obeying the equation (4.12). We then find the differential identities

$$
\begin{aligned}
& \mathbf{D}_{\hat{a}} F=\mathrm{i}\left(\Gamma_{\hat{a}}\right)_{\hat{\alpha}}^{\hat{\beta}} \eta_{\hat{\beta}}^{k} \epsilon_{k}^{\hat{\alpha}}+x_{\hat{a} \hat{b}} V^{\hat{b}}+G_{\hat{a} \hat{b}}^{k l} c_{k l}^{\hat{b}}, \\
& \mathbf{D}_{\hat{a}} V_{\hat{b}}=\mathrm{i} \eta_{\hat{a} \hat{b}} \eta^{\hat{\gamma} k} \epsilon_{\hat{\gamma} k}-2 \mathrm{i}\left(\Sigma_{\hat{a} \hat{b}}\right)^{\hat{\alpha} \hat{\beta}} \eta_{\hat{\alpha}}^{k} \epsilon_{\hat{\beta} k}-s_{k l} G_{\hat{a} \hat{b}}^{k l}-\frac{1}{2} x_{\hat{a} \hat{b}} F \\
& -\frac{1}{8} \varepsilon_{\hat{a} \hat{b} \hat{c} \hat{d} \hat{e}} n^{\hat{c} \hat{d}} V^{\hat{e}}+\frac{1}{4} \varepsilon_{\hat{a} \hat{b} \hat{c} \hat{d} \hat{e}} c^{\hat{c} k l} G^{\hat{d} \hat{e}}{ }_{k l}, \\
& \mathbf{D}_{\hat{a}} G_{\hat{b} \hat{c}}^{i j}=\frac{\mathrm{i}}{2} \varepsilon_{\hat{a} \hat{b} \hat{c} \hat{d} \hat{e}}\left(\Sigma^{\hat{d} \hat{e}}\right)^{\hat{\alpha} \hat{\beta}} \eta_{\hat{\alpha}}^{(i} \epsilon_{\hat{\beta}}^{j)}-\mathrm{i} \eta_{\hat{a}[\hat{b}}\left(\Gamma_{\hat{c}]}\right)^{\hat{\alpha} \hat{\beta}} \eta_{\hat{\alpha}}^{(i} \epsilon_{\hat{\beta}}^{j)} \\
& -\frac{1}{2} \varepsilon_{\hat{a} \hat{b} \hat{c} \hat{c} \hat{e}} s^{(i}{ }_{k} G^{\hat{d} \hat{e} j) k}-\frac{1}{2} \eta_{\hat{a}[\hat{b}} s^{i j} V_{\hat{c}]} \\
& -\frac{1}{2} \varepsilon_{\hat{d} \hat{e} \hat{f} \hat{b} \hat{c}} x_{\hat{a}}^{\hat{d}} G^{\hat{e} \hat{f} i j}+\frac{1}{2} \varepsilon_{\hat{d} \hat{e} \hat{f} \hat{a}[\hat{b}} n^{\hat{d} \hat{e}} G_{\hat{c}]} \hat{f}^{\hat{f}} i j \\
& -c_{[\hat{b}}\left({ }_{k} G_{\hat{c}] \hat{a}}{ }^{j) k}+\eta_{\hat{a}[\hat{b}} c^{\hat{d}\left({ }_{k}{ }_{k} G_{\hat{c}]} j\right) k}\right. \\
& +\frac{1}{2} \eta_{\hat{a}[\hat{b}} c_{\hat{c}]}^{i j} F+\frac{1}{8} \varepsilon_{\hat{a} \hat{b} \hat{c} \hat{d} \hat{e}} e^{\hat{d} c^{i j}} V^{\hat{e}} \text {. }
\end{aligned}
$$


These imply

$$
\begin{aligned}
\hat{\mathbf{D}}_{(\hat{a}} V_{\hat{b})} & =\frac{1}{5} \eta_{\hat{a} \hat{b}} \hat{\mathbf{D}}^{\hat{c}} V_{\hat{c}}, \\
\hat{\mathbf{D}}_{(\hat{a}} G_{\hat{b}) \hat{c}}{ }^{i j} & =-\frac{1}{4} \eta_{\hat{a} \hat{b}} \hat{\mathbf{D}}^{\hat{d}} G_{\hat{c} \hat{d}}{ }^{i j}+\frac{1}{4} \eta_{\hat{c}(\hat{a}} \hat{\mathbf{D}}^{\hat{d}} G_{\hat{b}) \hat{d}}{ }^{i j},
\end{aligned}
$$

where $\hat{\mathbf{D}}_{\hat{a}}$ denotes the covariant derivative (4.15). Eq. (B.5a) is equivalent to the conformal Killing equation (4.18).

Now let us restrict $\epsilon_{\hat{\alpha}}^{i}$ to be a Killing spinor, and hence $\eta_{k}^{\hat{\alpha}}=0$. Then we have

$$
\begin{aligned}
& \mathbf{D}_{\hat{a}} F=x_{\hat{a} \hat{b}} V^{\hat{b}}+G_{\hat{a} \hat{b}}{ }^{k l} c^{\hat{b}}{ }_{k l} \quad \Longrightarrow \quad V^{\hat{a}} \mathbf{D}_{\hat{a}} F=0, \\
& \mathbf{D}_{\hat{a}} V_{\hat{b}}=-s^{k l} G_{\hat{a} \hat{b} k l}-\frac{1}{2} x_{\hat{a} \hat{b}} F-\frac{1}{8} \varepsilon_{\hat{a} \hat{b} \hat{c} \hat{d} \hat{e}} n^{\hat{c} \hat{d}} V^{\hat{e}}+\frac{1}{4} \varepsilon_{\hat{a} \hat{b} \hat{c} \hat{d} \hat{e}} c^{\hat{c} k l} G^{\hat{d} \hat{e}} k l,
\end{aligned}
$$

and therefore $V^{\hat{a}}$ is a Killing vector field, eq. (4.24). Relation (B.5b) turns into

$$
\begin{aligned}
& \mathbf{D}_{\hat{a}} G_{\hat{b} \hat{c}}{ }^{i j}=-\frac{1}{2} \varepsilon_{\hat{a} \hat{b} \hat{c} \hat{d} \hat{e}} s^{(i}{ }_{k} G^{\hat{d e ̂} j) k}-\frac{1}{2} \eta_{\hat{a}[\hat{b}} s^{i j} V_{\hat{c}]} \\
& -\frac{1}{2} \varepsilon_{\hat{d} \hat{e} \hat{f} \hat{b} \hat{c}} x_{\hat{a}}^{\hat{d}} G^{\hat{e} \hat{f} i j}+\frac{1}{2} \varepsilon_{\hat{d} \hat{e} \hat{f} \hat{a}[\hat{b}} n^{\hat{d} \hat{e}} G_{\hat{c}]} \hat{f}^{\hat{f} i j}
\end{aligned}
$$

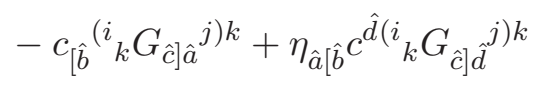

$$
\begin{aligned}
& +\frac{1}{2} \eta_{\hat{a}[\hat{b}} c_{\hat{c}]}^{i j} F+\frac{1}{8} \varepsilon_{\hat{a} \hat{b} \hat{c} \hat{d} \hat{e}} c^{\hat{d} i j} V^{\hat{e}} .
\end{aligned}
$$

The last result implies

$$
\begin{aligned}
\mathbf{D}_{[\hat{a}} G_{\hat{b} \hat{c}]}{ }^{i j}= & -\frac{1}{2} \varepsilon_{\hat{a} \hat{b} \hat{c} \hat{d} \hat{e}} s^{(i}{ }_{k} G^{\hat{d} \hat{e} j) k}+\frac{1}{8} \varepsilon_{\hat{a} \hat{b} \hat{c} \hat{d} \hat{e}} c^{\hat{d} i j} V^{\hat{e}}-c_{\left[\hat{a}{ }_{k} G_{\hat{b} \hat{c}]}{ }^{j}\right) k} \\
& +\frac{1}{2} \varepsilon_{\hat{d} \hat{e} \hat{f}[\hat{a} \hat{b}}\left(n^{\hat{d} \hat{e}}+x^{\hat{d} \hat{e}}\right) G_{\hat{c}]} \hat{f} i j
\end{aligned}
$$

and

$$
\hat{\mathbf{D}}^{\hat{a}} G_{\hat{a} \hat{b}}{ }^{i j}=-s^{i j} V_{\hat{b}}+c^{\hat{d}(i}{ }_{k} G_{\hat{c} \hat{d}}{ }^{j) k}+c_{\hat{c}}^{i j} F .
$$

Relation (B.7) dramatically simplifies if we are dealing with a supersymmetric solution of supergravity. In accordance with (9.13), we then have

$$
\mathbf{D}_{[\hat{a}} G_{\hat{b} \hat{c}]}{ }^{i j}=-\frac{1}{2} \varepsilon_{\hat{a} \hat{b} \hat{c} \hat{d} \hat{e}} s^{(i}{ }_{k} G^{\hat{d} \hat{e} j) k},
$$

where $s^{i j}=\frac{1}{2} \chi h^{i j}$ is covariantly constant. In the case of Poincaré supergravity, $\chi=0$ and the right-hand side of (B.9) vanishes. Thus the three two-forms $G^{i j}:=\frac{1}{2} G_{\hat{a} \hat{b}}{ }^{i j} e^{\hat{a}} e^{\hat{b}}$ are closed, ${ }^{18}$

$$
\mathrm{d} G^{i j}=0
$$

If the Killing vector $V^{\hat{a}}$ is time-like, the closed two-forms $G^{i j}$ turn out to define a hyperKähler structure on a $4 \mathrm{D}$ submanifold orthogonal to the orbit of $V^{\hat{a}}$ [51].

\footnotetext{
${ }^{18}$ Since the $\mathrm{SU}(2)$ curvature vanishes on-shell in Poincaré supergravity, the $\mathrm{SU}(2)$ connection can be completely gauged away.
} 
In the case of anti-de Sitter supergravity, $\chi \neq 0$, we may introduce a two-form $G:=$ $s_{i j} G^{i j}$. In accordance with (B.9), it is closed,

$$
\mathrm{d} G=0
$$

From eq. (B.2e) we also deduce

$$
G_{\hat{a}}^{\hat{c}} G_{\hat{c}}^{\hat{b}}=-\frac{1}{4} \chi^{2}\left(\delta_{\hat{a}}^{\hat{b}} F^{2}+V_{\hat{a}} V^{\hat{b}}\right) .
$$

If the Killing vector $V^{\hat{a}}$ is time-like, the closed two-form $G$ proves to define a Kähler structure on a $4 \mathrm{D}$ submanifold orthogonal to the orbit of $V^{\hat{a}}$ [59].

Open Access. This article is distributed under the terms of the Creative Commons Attribution License (CC-BY 4.0), which permits any use, distribution and reproduction in any medium, provided the original author(s) and source are credited.

\section{References}

[1] S.M. Kuzenko and G. Tartaglino-Mazzucchelli, Five-dimensional superfield supergravity, Phys. Lett. B 661 (2008) 42 [arXiv: 0710.3440] [INSPIRE].

[2] S.M. Kuzenko and G. Tartaglino-Mazzucchelli, $5 D$ supergravity and projective superspace, JHEP 02 (2008) 004 [arXiv:0712.3102] [INSPIRE].

[3] S.M. Kuzenko and G. Tartaglino-Mazzucchelli, Super-Weyl invariance in $5 D$ supergravity, JHEP 04 (2008) 032 [arXiv:0802.3953] [INSPIRE].

[4] M. Zucker, Minimal off-shell supergravity in five-dimensions, Nucl. Phys. B 570 (2000) 267 [hep-th/9907082] [INSPIRE].

[5] M. Zucker, Gauged N=2 off-shell supergravity in five-dimensions, JHEP 08 (2000) 016 [hep-th/9909144] [INSPIRE].

[6] M. Zucker, Off-shell supergravity in five-dimensions and supersymmetric brane world scenarios, Fortsch. Phys. 51 (2003) 899 [INSPIRE].

[7] T. Kugo and K. Ohashi, Supergravity tensor calculus in $5 D$ from $6 D$, Prog. Theor. Phys. 104 (2000) 835 [hep-ph/0006231] [INSPIRE].

[8] T. Kugo and K. Ohashi, Off-shell $d=5$ supergravity coupled to matter Yang-Mills system, Prog. Theor. Phys. 105 (2001) 323 [hep-ph/0010288] [INSPIRE].

[9] T. Fujita and K. Ohashi, Superconformal tensor calculus in five-dimensions, Prog. Theor. Phys. 106 (2001) 221 [hep-th/0104130] [InSPIRE].

[10] E. Bergshoeff et al., Weyl multiplets of $N=2$ conformal supergravity in five-dimensions, JHEP 06 (2001) 051 [hep-th/0104113] [INSPIRE].

[11] E. Bergshoeff et al., Superconformal $N=2, D=5$ matter with and without actions, JHEP 10 (2002) 045 [hep-th/0205230] [INSPIRE].

[12] E. Bergshoeff et al., $N=2$ supergravity in five-dimensions revisited, Class. Quant. Grav. 21 (2004) 3015 [hep-th/0403045] [INSPIRE]. 
[13] P.S. Howe, Off-shell N=2 and N=4 supergravity in five-dimensions, in: Quantum Structure of Space and Time, M.J. Duff and C.J. Isham eds., Cambridge University Press, Cambridge U.K. (1982), pg. 239.

[14] P.S. Howe and U. Lindström, The supercurrent in five-dimensions, Phys. Lett. B 103 (1981) 422 [inSPIRE].

[15] S.M. Kuzenko, On compactified harmonic/projective superspace, $5 D$ superconformal theories and all that, Nucl. Phys. B 745 (2006) 176 [hep-th/0601177] [INSPIRE].

[16] U. Lindström and M. Roček, New hyperKähler metrics and new supermultiplets, Commun. Math. Phys. 115 (1988) 21 [InSPIRE].

[17] U. Lindström and M. Roček, $N=2$ super Yang-Mills theory in projective superspace, Commun. Math. Phys. 128 (1990) 191 [INSPIRE].

[18] S.M. Kuzenko and G. Tartaglino-Mazzucchelli, Five-dimensional $N=1$ AdS superspace: geometry, off-shell multiplets and dynamics, Nucl. Phys. B 785 (2007) 34 [arXiv: 0704.1185] [INSPIRE].

[19] I.L. Buchbinder and S.M. Kuzenko, Ideas and Methods of Supersymmetry and Supergravity or a Walk Through Superspace, IOP, Bristol U.K. (1995).

[20] S.M. Kuzenko and G. Tartaglino-Mazzucchelli, Field theory in $4 D N=2$ conformally flat superspace, JHEP 10 (2008) 001 [arXiv:0807.3368] [inSPIRE].

[21] D. Butter and S.M. Kuzenko, $N=2$ supersymmetric $\sigma$-models in AdS, Phys. Lett. B 703 (2011) 620 [arXiv:1105.3111] [InSPIRE].

[22] D. Butter and S.M. Kuzenko, The structure of $N=2$ supersymmetric nonlinear $\sigma$-models in $A d S_{4}, J H E P 11$ (2011) 080 [arXiv:1108.5290] [INSPIRE].

[23] D. Butter, S.M. Kuzenko, U. Lindström and G. Tartaglino-Mazzucchelli, Extended supersymmetric $\sigma$-models in AdS 4 from projective superspace, JHEP 05 (2012) 138 [arXiv: 1203.5001] [INSPIRE].

[24] S.M. Kuzenko and G. Tartaglino-Mazzucchelli, Three-dimensional $N=2$ (AdS) supergravity and associated supercurrents, JHEP 12 (2011) 052 [arXiv:1109.0496] [INSPIRE].

[25] S.M. Kuzenko, U. Lindström and G. Tartaglino-Mazzucchelli, Three-dimensional $(p, q)$ AdS superspaces and matter couplings, JHEP 08 (2012) 024 [arXiv:1205.4622] [INSPIRE].

[26] D. Butter, S.M. Kuzenko and G. Tartaglino-Mazzucchelli, Nonlinear $\sigma$-models with AdS supersymmetry in three dimensions, JHEP 02 (2013) 121 [arXiv:1210.5906] [INSPIRE].

[27] G. Festuccia and N. Seiberg, Rigid supersymmetric theories in curved superspace, JHEP 06 (2011) 114 [arXiv: 1105.0689] [INSPIRE].

[28] B. Jia and E. Sharpe, Rigidly supersymmetric gauge theories on curved superspace, JHEP 04 (2012) 139 [arXiv:1109.5421] [INSPIRE].

[29] H. Samtleben and D. Tsimpis, Rigid supersymmetric theories in 4d Riemannian space, JHEP 05 (2012) 132 [arXiv: 1203.3420] [INSPIRE].

[30] C. Klare, A. Tomasiello and A. Zaffaroni, Supersymmetry on curved spaces and holography, JHEP 08 (2012) 061 [arXiv: 1205.1062] [INSPIRE].

[31] T.T. Dumitrescu, G. Festuccia and N. Seiberg, Exploring curved superspace, JHEP 08 (2012) 141 [arXiv:1205.1115] [INSPIRE]. 
[32] C. Closset, T.T. Dumitrescu, G. Festuccia, Z. Komargodski and N. Seiberg, Contact terms, unitarity and F-maximization in three-dimensional superconformal theories, JHEP 10 (2012) 053 [arXiv: 1205.4142] [INSPIRE].

[33] C. Closset, T.T. Dumitrescu, G. Festuccia, Z. Komargodski and N. Seiberg, Comments on Chern-simons contact terms in three dimensions, JHEP 09 (2012) 091 [arXiv:1206.5218] [INSPIRE].

[34] D. Cassani, C. Klare, D. Martelli, A. Tomasiello and A. Zaffaroni, Supersymmetry in Lorentzian curved spaces and holography, Commun. Math. Phys. 327 (2014) 577 [arXiv:1207.2181] [INSPIRE].

[35] J.T. Liu, L.A. Pando Zayas and D. Reichmann, Rigid supersymmetric backgrounds of minimal off-shell supergravity, JHEP 10 (2012) 034 [arXiv:1207.2785] [INSPIRE].

[36] T.T. Dumitrescu and G. Festuccia, Exploring curved superspace (II), JHEP 01 (2013) 072 [arXiv: 1209.5408] [INSPIRE].

[37] A. Kehagias and J.G. Russo, Global supersymmetry on curved spaces in various dimensions, Nucl. Phys. B 873 (2013) 116 [arXiv:1211.1367] [INSPIRE].

[38] C. Closset, T.T. Dumitrescu, G. Festuccia and Z. Komargodski, Supersymmetric field theories on three-manifolds, JHEP 05 (2013) 017 [arXiv:1212.3388] [INSPIRE].

[39] K. Hristov, A. Tomasiello and A. Zaffaroni, Supersymmetry on three-dimensional Lorentzian curved spaces and black hole holography, JHEP 05 (2013) 057 [arXiv:1302.5228] [INSPIRE].

[40] P. de Medeiros and S. Hollands, Conformal symmetry superalgebras, Class. Quant. Grav. 30 (2013) 175016 [arXiv: 1302.7269] [INSPIRE].

[41] P. de Medeiros and S. Hollands, Superconformal quantum field theory in curved spacetime, Class. Quant. Grav. 30 (2013) 175015 [arXiv:1305.0499] [INSPIRE].

[42] C. Closset, T.T. Dumitrescu, G. Festuccia and Z. Komargodski, The geometry of supersymmetric partition functions, JHEP 01 (2014) 124 [arXiv:1309.5876] [INSPIRE].

[43] N.S. Deger, A. Kaya, H. Samtleben and E. Sezgin, Supersymmetric warped AdS in extended topologically massive supergravity, Nucl. Phys. B 884 (2014) 106 [arXiv:1311.4583] [INSPIRE].

[44] C. Closset and S. Cremonesi, Comments on $\mathcal{N}=(2,2)$ supersymmetry on two-manifolds, JHEP 07 (2014) 075 [arXiv:1404.2636] [INSPIRE].

[45] S.M. Kuzenko, Symmetries of curved superspace, JHEP 03 (2013) 024 [arXiv:1212.6179] [INSPIRE].

[46] S.M. Kuzenko, U. Lindström, M. Roček, I. Sachs and G. Tartaglino-Mazzucchelli, Three-dimensional $N=2$ supergravity theories: from superspace to components, Phys. Rev. D 89 (2014) 085028 [arXiv:1312.4267] [INSPIRE].

[47] S.M. Kuzenko, U. Lindström and G. Tartaglino-Mazzucchelli, Off-shell supergravity-matter couplings in three dimensions, JHEP 03 (2011) 120 [arXiv:1101.4013] [INSPIRE].

[48] S.M. Kuzenko and W.D. Linch III, On five-dimensional superspaces, JHEP 02 (2006) 038 [hep-th/0507176] [INSPIRE].

[49] S.M. Kuzenko and G. Tartaglino-Mazzucchelli, Conformally flat supergeometry in five dimensions, JHEP 06 (2008) 097 [arXiv: 0804.1219] [INSPIRE]. 
[50] D.Z. Freedman and A. Van Proeyen, Supergravity, Cambridge University Press, Cambridge U.K. (2012).

[51] J.P. Gauntlett, J.B. Gutowski, C.M. Hull, S. Pakis and H.S. Reall, All supersymmetric solutions of minimal supergravity in five-dimensions, Class. Quant. Grav. 20 (2003) 4587 [hep-th/0209114] [INSPIRE].

[52] H. Stephani, D. Kramer, M. MacCullum, C. Hoenselaers and E. Hertl, Exact Solutions of Einstein's Field Equations, second edition, Cambridge University Press, Cambridge U.K. (2003).

[53] T. Ortin, Gravity and Strings, Cambridge University Press, Cambridge U.K. (2004).

[54] B. de Wit, R. Philippe and A. Van Proeyen, The improved tensor multiplet in $N=2$ supergravity, Nucl. Phys. B 219 (1983) 143 [INSPIRE].

[55] U. Lindström and M. Roček, Scalar tensor duality and $N=1, N=2$ Nonlinear $\sigma$-models, Nucl. Phys. B 222 (1983) 285 [INSPIRE].

[56] D. Butter and S.M. Kuzenko, New higher-derivative couplings in $4 D N=2$ supergravity, JHEP 03 (2011) 047 [arXiv: 1012.5153] [INSPIRE].

[57] D. Butter and S.M. Kuzenko, $N=2$ AdS supergravity and supercurrents, JHEP 07 (2011) 081 [arXiv: 1104.2153] [INSPIRE].

[58] S.M. Kuzenko and S. Theisen, Correlation functions of conserved currents in $N=2$ superconformal theory, Class. Quant. Grav. 17 (2000) 665 [hep-th/9907107] [INSPIRE].

[59] J.P. Gauntlett and J.B. Gutowski, All supersymmetric solutions of minimal gauged supergravity in five-dimensions, Phys. Rev. D 68 (2003) 105009 [Erratum ibid. D 70 (2004) 089901] [hep-th/0304064] [INSPIRE].

[60] J. Bagger and C. Xiong, AdS $S_{5}$ supersymmetry in $N=1$ superspace, JHEP 07 (2011) 119 [arXiv: 1105.4852] [INSPIRE].

[61] J. Bagger and J. Li, Supersymmetric nonlinear $\sigma$-model in $A d S_{5}$, Phys. Lett. B 702 (2011) 291 [arXiv:1106.2343] [INSPIRE].

[62] N.J. Hitchin, A. Karlhede, U. Lindström and M. Roček, HyperKähler metrics and supersymmetry, Commun. Math. Phys. 108 (1987) 535 [INSPIRE].

[63] L. Álvarez-Gaumé and D.Z. Freedman, Geometrical structure and ultraviolet finiteness in the supersymmetric $\sigma$-model, Commun. Math. Phys. 80 (1981) 443 [INSPIRE].

[64] C.M. Hull, A. Karlhede, U. Lindström and M. Roček, Nonlinear $\sigma$ models and their gauging in and out of superspace, Nucl. Phys. B 266 (1986) 1 [INSPIRE].

[65] S.M. Kuzenko, Projective superspace as a double punctured harmonic superspace, Int. J. Mod. Phys. A 14 (1999) 1737 [hep-th/9806147] [inSPIRE].

[66] S.J. Gates Jr. and S.M. Kuzenko, The CNM hypermultiplet nexus, Nucl. Phys. B 543 (1999) 122 [hep-th/9810137] [INSPIRE].

[67] S.J. Gates Jr. and S.M. Kuzenko, $4 D, N=2$ supersymmetric off-shell $\sigma$-models on the cotangent bundles of Kähler manifolds, Fortsch. Phys. 48 (2000) 115 [hep-th/9903013] [INSPIRE].

[68] D. Kaledin, Hyperkähler structures on total spaces of holomorphic cotangent bundles, in Hyperkähler Manifolds, D. Kaledin and M. Verbitsky eds., International Press, Cambridge U.S.A. (1999) [alg-geom/9710026]. 
[69] D. Kaledin, A canonical hyperkähler metric on the total space of a cotangent bundle, in Quaternionic Structures in Mathematics and Physics, S. Marchiafava, P. Piccinni and M. Pontecorvo eds., World Scientific, Singapore (2001) [alg-geom/0011256].

[70] B. Feix, Hyperkähler metrics on cotangent bundles, J. Reine Angew. Math. 532 (2001) 33.

[71] Y. Pan, Rigid supersymmetry on 5-dimensional Riemannian manifolds and contact geometry, JHEP 05 (2014) 041 [arXiv: 1308.1567] [INSPIRE].

[72] Y. Imamura and H. Matsuno, Supersymmetric backgrounds from $5 d \mathcal{N}=1$ supergravity, JHEP 07 (2014) 055 [arXiv: 1404.0210] [INSPIRE].

[73] S. Deser, Scale invariance and gravitational coupling, Annals Phys. 59 (1970) 248 [INSPIRE].

[74] S.M. Kuzenko, Symmetries of curved superspace, talk given at the Second ANZAMP Meeting, Mooloolaba Australia, 27-29 November 2013. 